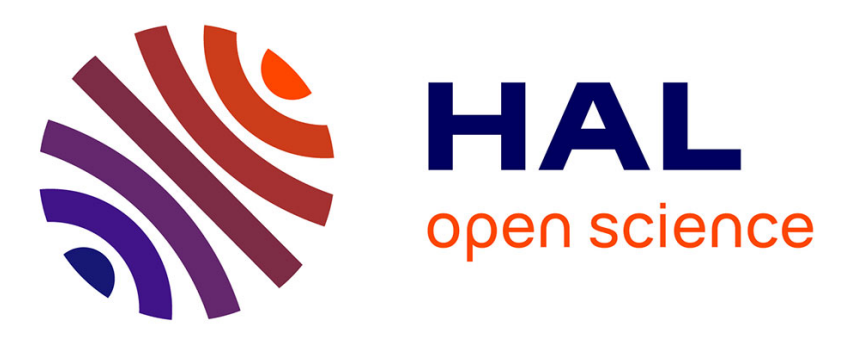

\title{
Reverberation Chambers à la carte: An overview of the different mode-stirring techniques
}

\author{
Ramiro Serra, Andy C. Marvin, Franco Moglie, Walter Mariani Primiani, \\ Andrea Cozza, Luk R. Arnaut, Yi Huang, Mike O. Hatfield, Marco Klingler, \\ Frank Leferink
}

\section{To cite this version:}

Ramiro Serra, Andy C. Marvin, Franco Moglie, Walter Mariani Primiani, Andrea Cozza, et al.. Reverberation Chambers à la carte: An overview of the different mode-stirring techniques. Electromagnetic Compatibility Magazine, IEEE, 2017, 6 (1), pp.63-78. 10.1109/MEMC.2017.7931986 . hal-01440203

\section{HAL Id: hal-01440203 \\ https://hal-centralesupelec.archives-ouvertes.fr/hal-01440203}

Submitted on 26 Jan 2017

HAL is a multi-disciplinary open access archive for the deposit and dissemination of scientific research documents, whether they are published or not. The documents may come from teaching and research institutions in France or abroad, or from public or private research centers.
L'archive ouverte pluridisciplinaire HAL, est destinée au dépôt et à la diffusion de documents scientifiques de niveau recherche, publiés ou non, émanant des établissements d'enseignement et de recherche français ou étrangers, des laboratoires publics ou privés. 


\section{Reverberation Chambers à la carte: An overview of the different mode-stirring techniques}

R. Serra, A. C. Marvin, F. Moglie, V. Mariani Primiani, A. Cozza, L. R. Arnaut, Y. Huang, M. O. Hatfield, M. Klingler, F. Leferink.

\section{Introduction}

Reverberation chambers ( $\mathrm{RC}$ ), a name inspired in room acoustics, are also known in literature as reverberating, reverb, mode-stirred or mode-tuned chambers. In their basic form, they consist of a shielded metallic enclosure, forming a cavity resonator, together with some mode-stirring mechanism. The main goal of such stirring mechanism is to generate an amplitude-varying electromagnetic field that is ideally statistically uniform.

A typical RC facility is shown in Fig. 1, featuring, in this case, a rotating stirrer, a field generating antenna which injects electromagnetic (EM) energy inside the cavity and a receiving antenna for field monitoring.

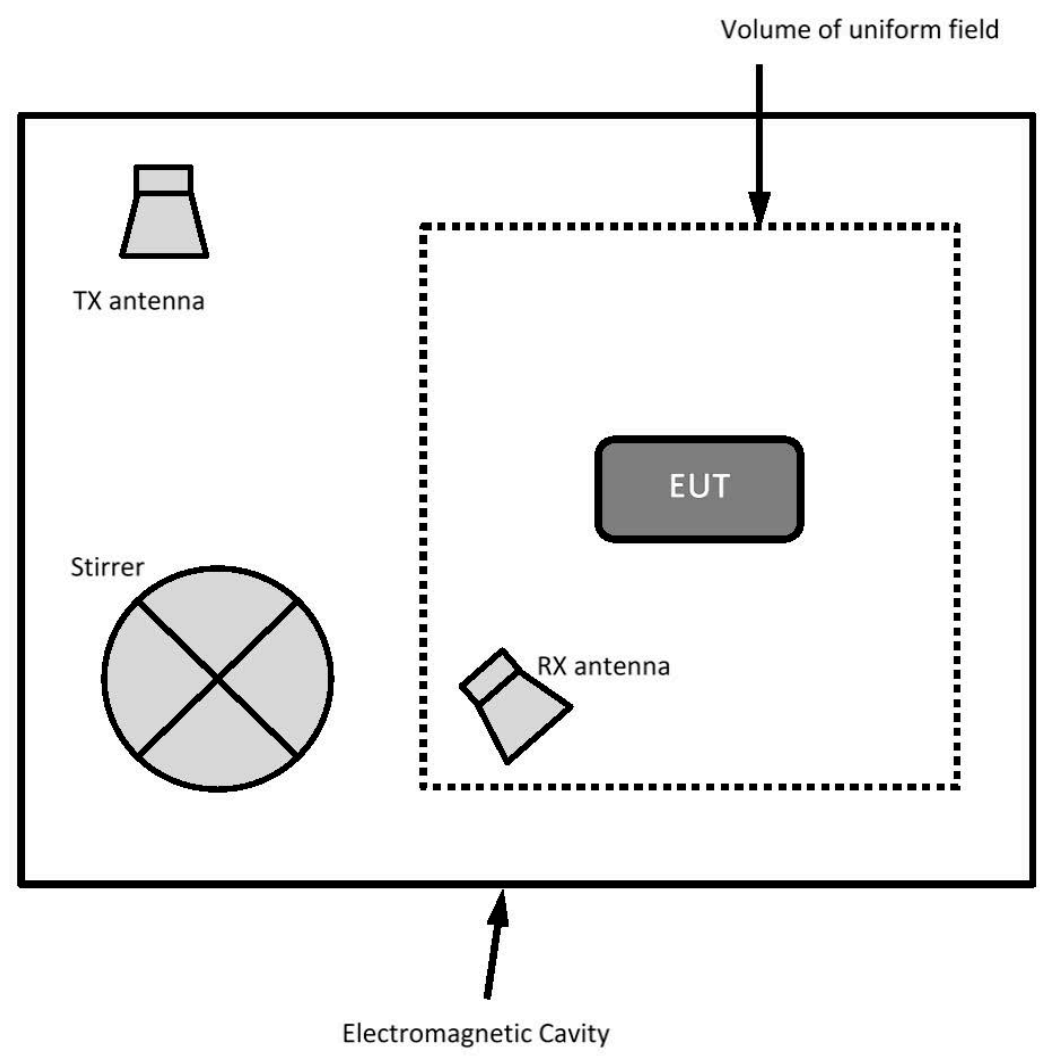

Fig. 1. Typical RC facility. 
The volume of uniform field, referred in Fig. 1, is also known as the working volume (WV) and it represents the region inside the chamber where statistical field uniformity is achieved.

Statistical field uniformity implies:

1) Homogeneity: uniformity w.r.t. location (spatial uniformity).

2) Isotropy: uniformity w.r.t. orientation (polarization uniformity).

Should a particular stirring mechanism be a successful one, the two abovementioned characteristics of homogeneity and isotropy are fulfilled inside a RC.

What kind of stirring mechanisms can perform successful stirring? Over the years, many different strategies have been designed and several of them implemented. This paper recollects the most relevant ones and provides an overview of them.

\section{Statistical field uniformity}

The concept of statistical field uniformity for RCs should be understood differently to the concept of field uniformity normally used in other test environments like, for instance, open area test sites, anechoic chambers or (G)TEM cells. In those environments, the field is uniform when it is "the same everywhere at any moment", while a statistically uniform field in a RC is found if "on average, and within an acceptable uncertainty, the stirred vector field (and its spatial orientation, magnitude and intensity) is the same at different spatial locations". Yet the fields at sufficiently separated locations within the WV and different orientations will be widely different, for any given stir state (e.g. a fixed angular position of the stirrer), its statistical parameters (e.g., mean, standard deviation, etc.) are the same everywhere, after completion of a full stirring cycle.

This statistical description of fields might often be somehow complicated for EMC engineers who are used to deterministic fields, like the ones present in open area test sites, etc. As an illustrative example of how a statistically uniform field should be understood, Fig. 2 shows the power received by an antenna when a field is generated inside a RC through the transmitting antenna. The variations in the received field are caused by the rotation of the stirrer inside this particular RC. At each change of the angular position of the stirrer, the EM field distribution inside the RC changes (changing boundary conditions). The received power in Fig. 2 is recorded for two different locations an orientations of the receiving antenna inside the WV. It must be observed that the field at one position is different in amplitude to the field at the other position for each angle of the stirrer. Nevertheless, if we compare the statistical properties of the field at both positions after a full stirring cycle, summarized in Table I, we can see that the fields look (statistically) very similar to one another.

This statistical uniformity does not happen anywhere inside the RC, but rather in the WV alone which is often defined as the volume whose boundaries are sufficiently far away (typically a quarter of a wavelength at the lowest frequency of operation) from the cavity walls, antennas, stirrer(s) and from any other electromagnetically relevant object inside the RC. 
The usefulness of such a statistically uniform field in, for instance, immunity tests is evident. The equipment under test (EUT) would be subject to the same field (statistically) regardless of its location and orientation inside the chamber. Reciprocity helps us understand that an analogous situation can be generated to assure proper emission tests where the receiving antenna would receive the same field (statistically) being emitted by the EUT, regardless of its location and orientation inside the chamber. This aspect of statistical field uniformity represents a major advantage for EMC tests.

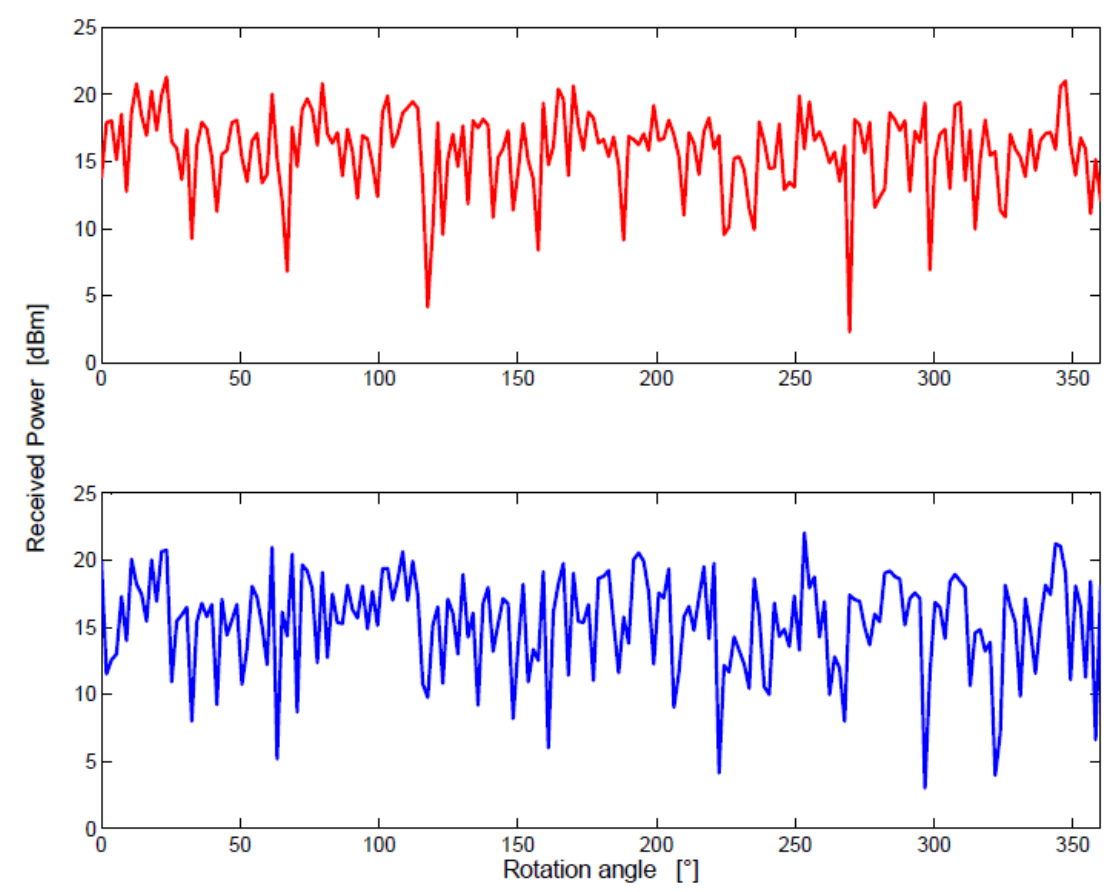

Fig. 2. Powers received by an antenna at $1 \mathrm{GHz}$ as a function of the rotation angle of the stirrer for two different positions inside a RC.

Table I. Statistical properties of the two sets of data of Fig. 1 measured at different locations and orientations within a RC.

\begin{tabular}{|l|l|l|}
\hline & Position 1 & Position 2 \\
\hline Maximum value [dBm] & 21.3 & 22.0 \\
\hline Mean value [dBm] & 16.6 & 16.6 \\
\hline Maximum to mean ratio [dB] & 4.7 & 5.4 \\
\hline Maximum to minimum ratio [dB] & 19.0 & 19.0 \\
\hline Standard deviation [dBm] & 14.2 & 14.8 \\
\hline
\end{tabular}

First things first: we need a resonant cavity 
A RC can be realized only if we firstly count with an electromagnetic cavity resonator. This is usually achieved by an enclosure with conducting walls typically, though not exclusively, of rectangular shape. When energy is injected inside such a closed cavity by means of e.g. a transmitting antenna, then infinite resonant modes are excited. At each frequency of excitation, only a finite number of these modes significantly contributes to the total field inside the cavity. The higher the frequency of excitation the higher the number of contributing modes. The total field distribution is then found by the superposition of all the resonant modes significantly contributing inside the cavity. Such superposition of resonant modes forms three dimensional standing wave patterns inside the chamber with regions where the field is relatively small and regions where it is relatively large.

An analogous situation can be found in musical acoustics. Every physical object has its own natural acoustic resonances like, for instance, a string or a glass bottle. The frequencies of resonance depend mainly on their physical properties such as, e.g. material, size or shape. The larger (or thicker) the string, the lower the fundamental frequencies of resonance. The higher the tension on the string, the higher the fundamental frequencies of resonance. These resonances are mathematically found by solving the homogeneous sound wave equation in such geometries. The homogeneous wave equation is nothing more than the wave equation in absence of a source. When we apply a source like, for instance, plucking the string with a finger, or blowing air inside the bottle, these objects will produce a sound which can be expressed as the (weighted) contribution of all the natural resonances. The mathematical procedure of expressing the solution as the weighted sum of all the present modes is often known as "modal expansion" [1]. As an example, Fig. 3 shows the first four resonant modes for a string fixed at both endings (called fixed-fixed string). When plucked, the compound sound can be found by summing all the excited modes with each expansion coefficient (mode weight). Figure 3 shows in the bottom curve the vibration of the string when plucked at a distance $1 / 3$ of the length (only the contribution of the first 9 modes was taken into account). 


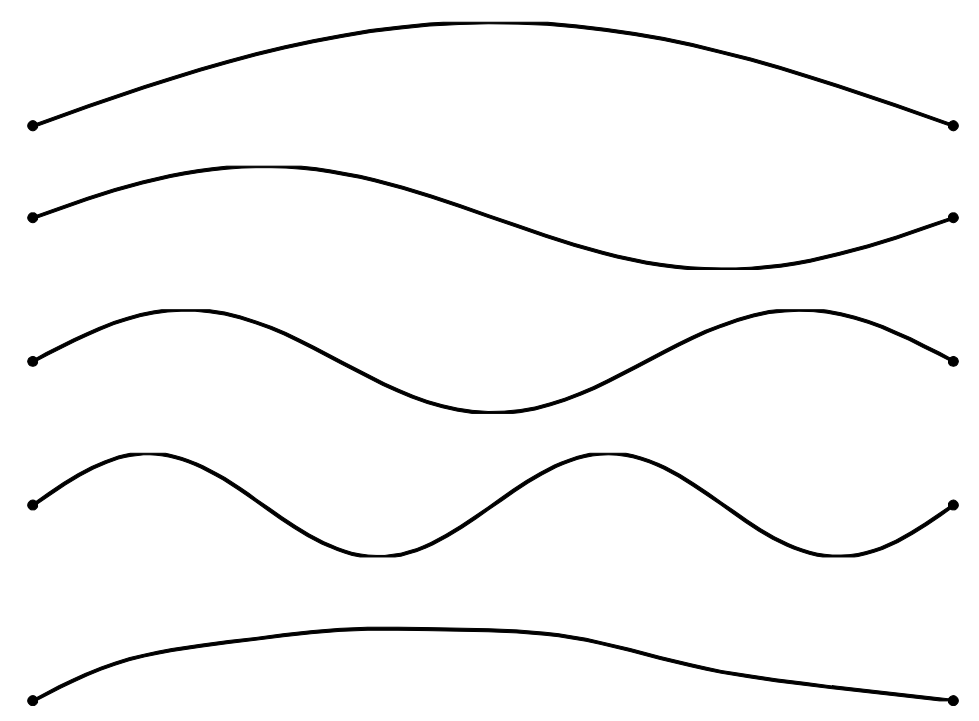

Fig. 3. An analogy to electromagnetic resonators: the vibration of fixed-fixed string. The fundamental and the first three overtones which form a harmonic series is illustrated by the upper fours curves. The bottom curve shows the standing wave vibration of the string plucked at a distance $1 / 3$ of its length.

Following the acoustics analogy, electromagnetic resonant modes are the natural (nontrivial) solutions of the homogeneous electromagnetic wave equation solved for the specific geometry under study.

\section{Not enough: a cavity resonator is not a reverberation chamber}

Understanding electromagnetic resonance is a basic and important step to understanding RCs. Nevertheless, this is not enough since a RC is not simply a cavity resonator. The standing wave patterns found in cavity resonators represent, clearly, a strong nonuniform distribution of the field, which is the opposite of what RCs are supposed to deliver i.e. a (statistically) uniform field, as stated in the Introduction. Therefore, the process of mode stirring becomes an essential constituent of RCs. Mode stirring is the procedure of changing the boundary conditions of the cavity and therefore producing also a change in the contributing resonant modes. Changing the source position, inserting a rotating metal paddle, or even building a cavity with flexible conducting material and make it vibrate, are some examples on how to stir the modes. At every stir state, the mode expansion and thus the field distribution will change.

Care must be taken in that not every change in the boundary conditions is a successful mode stirring technique per se. The modes must not only be stirred, they must be stirred well. The final goal of mode stirring is to create a statistical uniform field inside the working volume of the 
RC. Under a successful mode stirring strategy, the electromagnetic field inside any location within the working volume and at any orientation will present the same statistical properties.

Probably the most widespread method to assess field uniformity is the one specified in the international standard [2]. This measure of field uniformity combines, in one metric, field homogeneity and field isotropy. In this method, the RC is excited with a transmitting antenna and the electric field is monitored at the eight corners of the WV using a three-axial electric field probe while the stirring process takes place (e.g. a stirrer rotates). The standard deviation of the eight maximum recorded field values (for a complete stirring cycle) at each different location is then calculated per coordinate vector (i.e. $\sigma_{x}, \sigma_{y}$ and $\sigma_{z}$ ) and also for all the coordinate vectors combined (i.e. $\sigma_{24}$ or sometimes also known as $\sigma_{\text {tot }}$ ). The IEC limit for field nonuniformity is defined at $3 \mathrm{~dB}$ from the mean from $400 \mathrm{MHz}$ upward, ramping up linearly from $3 \mathrm{~dB}$ to $4 \mathrm{~dB}$ when the frequency is decreased from $400 \mathrm{MHz}$ to $100 \mathrm{MHz}$ [2] and it is $4 \mathrm{~dB}$ below $100 \mathrm{MHz}$. A more detailed description of how this metric is calculated can be found in the standard [2] and will also be discussed in the companion paper to this Theme paper (Part II).

We have been describing the mode-stirring process as the change of the boundary conditions in general. Very often, in practice, a distinction is made between mode-stirring and mode-tuning. Whenever the stirring process is applied continuously then the term "mode-stirring" is preferred, leaving the term "mode-tuning" for stirring processes that are implemented in a stepped fashion.

\section{We should thank the ergodic hypothesis.}

The term "ergodic" was invented in statistical physics and refers to those stationary dynamical systems on which any ensemble average is exchangeable with time average. Roughly this means that after a sufficiently long time every statistical characteristic of a set of states within the process will spread evenly over the entire space.

As an example of the above, consider the kinetic theory of gases. For a gas consisting of a number of molecules in a closed vessel, any particular statistical index describing a set of possible system states (e.g. the average distance from the center or the range of velocities of all molecules), remains invariant for all time on a confined region where (total) energy is constant. See Fig. 4 for a schematic representation. This basically allows to describe and predict the future of this process without knowing the exact formulas for the trajectories of all involved molecules. For example, if we want to know the average velocity (both its magnitude, i.e, speed, and its direction) of all the gas molecules within the vessel, we could either: (a) measure the velocity of all the molecules at an exact moment, or (b) "follow" one particular molecule and measure all its velocities as it collides with the other molecules, long time enough. Average in (b) will converge into the one of (a), due to the fact that we are dealing with an ergodic process.

It is important to note, however, that the ergodic hypothesis is, in fact, a hypothesis and therefore, remains an assumption. Whether or not might it be applicable to a specific process, is 
often subject to discussion. Luckily enough, there hasn't been any controversy against the ergodic hypothesis among RC users!

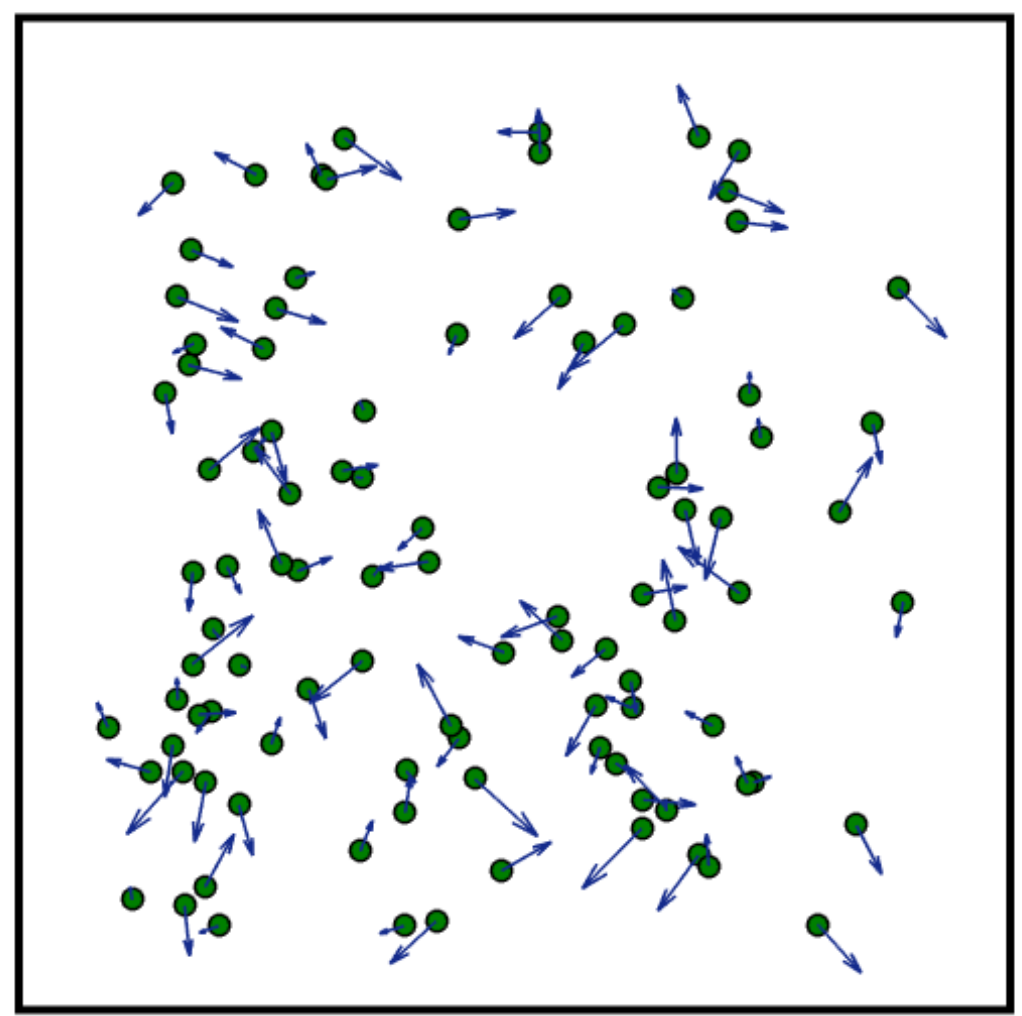

Fig. 4. A simple example of an ergodic process: gas molecules collisions in a closed vessel.

When applied to RCs, the ergodic hypothesis finds a good ground in a simple intuition, nicely explained in [3]. Assuming that the cavity is overmoded and each contributing mode is excited with (approximately) the same level, then the overlap of many contributing resonant modes at a given frequency of excitation, is equal to an aggregation (in time) of contributions from one single resonant mode when properly stirred.

The total field inside a resonant cavity can be expanded as an infinite sum of its natural resonant modes, which are a function of the shape, size, loss and dielectric properties of the walls and the medium filling the cavity. In turn, each one of these contributing modes is weighted (modulated) by a factor that depends on the source type, orientation, position, etc. Each one of the influencing factors in this field expansion is a candidate for mode-stirring. For instance, when a spatial change of the receiving antenna, a stirrer rotation or a frequency shift is considered, the probability distributions that characterize EM quantities are the same, assuming the ergodic hypothesis to hold. 
Therefore, it is all thanks to the ergodic hypothesis! It is the basic fundamental principle that allows so many different stirring techniques, either already proposed or waiting to be proposed...

\section{The early years}

Without calling it specifically a "reverberation chamber", nor either of its synonyms, the first reported test environment applied to shielding effectiveness (thus a typical EMC test), and formally coinciding with a RC dates back to 1971 and is found in the military standard MIL-STD 1377 [4]. Figure 5 shows the schematic for a shielding effectiveness test on a weapon cable to be tested.

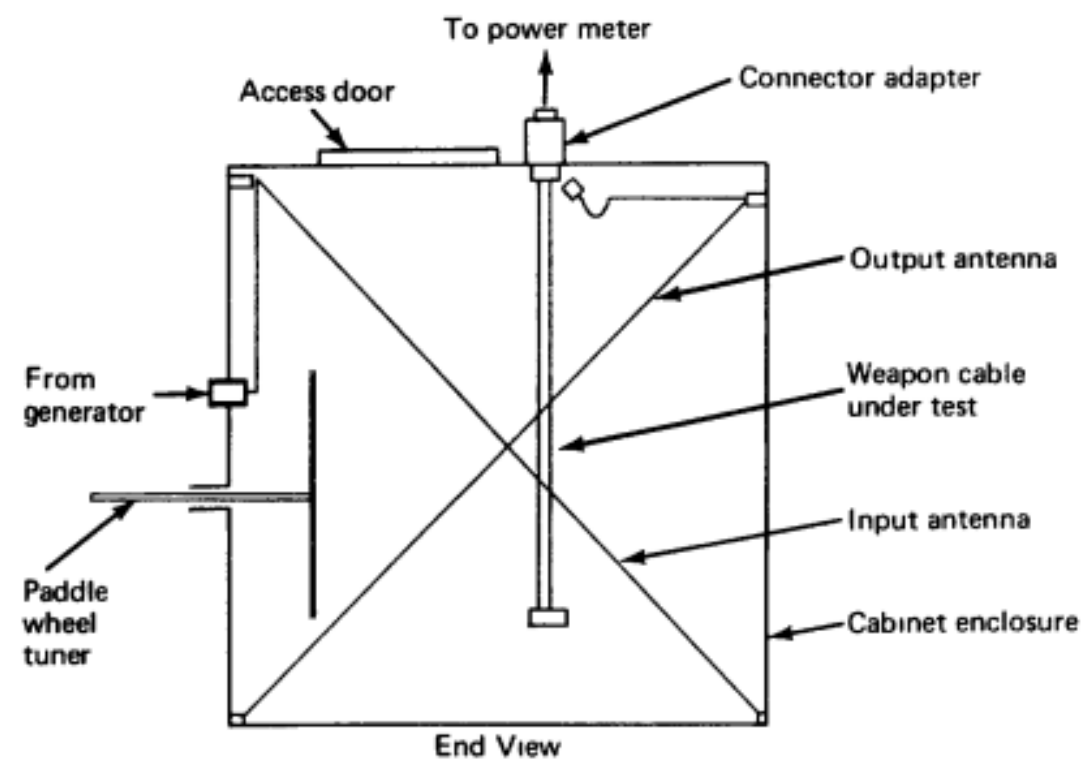

Fig. 5. First reported test setup formally coinciding with the main characteristics of a RC. Picture extracted from [4].

The "test cabinet", as it was named in [4], features all the main components of a RC, viz. a shielded enclosure and a stirring mechanism (the paddle wheel tuner).

In 1973, E. Paolini and L. Piccioli report on the use of a reverberation chamber [5] in interference assessment of ISM wireless devices. Yet, probably the most widely recognized (and cited) RC for use in EMC testing within the EMC community was developed and reported in 1976 by P. Corona and G. Latmiral at the Istituto Universitario Navale (IUN) in Naples, Italy. Corona and Latmiral set the path to a wider spectrum of EMC tests, including not only shielding effectiveness, but also immunity and emission. The original report on this RC was written in Italian [6] and shortly afterwards a paper was published on the IEEE Transactions on EMC [7]. Since then, RCs have been gaining increased popularity as an alternative test setup within the EMC community - and beyond. 
The RC described by P. Corona and G. Latmiral used two low-profile metallic rectangular vanes rotating parallel to two perpendicular walls in order to achieve the desired (statistical) field uniformity, quite similar to the paddle wheel of Fig. 5. Figure 6 shows this original sketch.

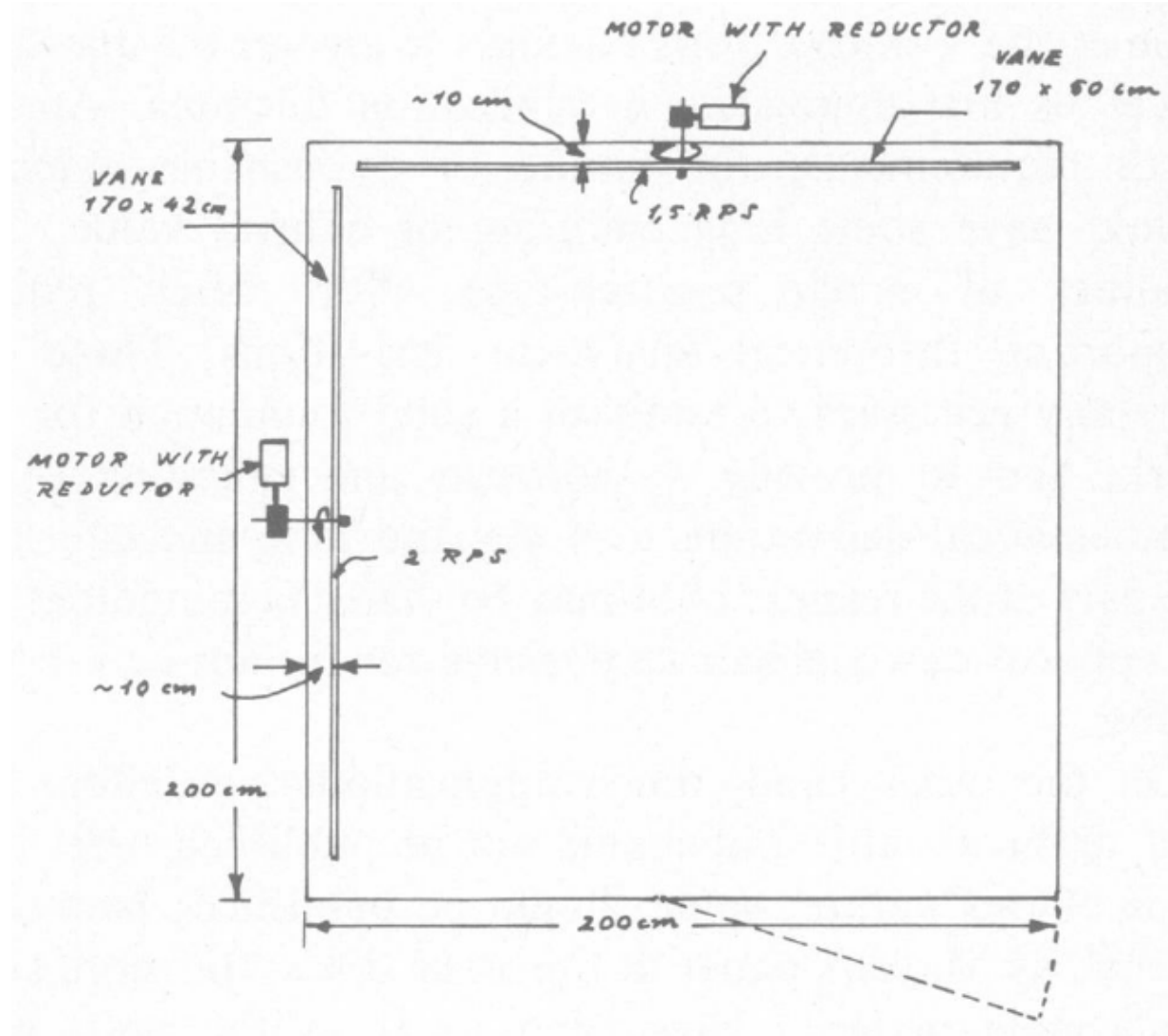

Fig. 6. Schematic of the '76 RC facility at Istituto Universitario Navale in Naples. Figure extracted from [6].

Further details on the early years research at IUN are present in several papers of the Special Issue honoring Paolo Corona (IEEE Transactions on EMC, vol. 58, issue 3, June 2016), particularly in [8].

Through subsequent years after the RC with rotating vanes of Fig. 6 , and accompanying the ever-increasing research and development activities on RCs, researchers interested in such chambers have proposed a vast number of different and alternative stirring techniques.

And what an interesting and exciting exercise of creativity this "stirring journey" has been!

This paper summarizes and overviews the main alternative stirring techniques proposed in the scientific literature during the last four decades. We believe that this list is still in evolution and further strategies for mode-stirring might become available in the future.

After these preliminaries, in the next sections we dive into all the different stirring techniques, explain their basic characteristics and, where possible, show their performance. The stirring 
techniques have been divided into two main groups: mechanical mode-stirring techniques and electronic mode-stirring techniques.

\section{Mechanical Mode Stirring.}

We denominate "mechanical" stirring to all those techniques that make use of movements, i.e. translations, rotations, vibrations, etc. of a RC constituent like a large metallic scatterer or a wall. The RC in [6] clearly falls in this category due to the rotating vanes.

We identify two subclasses of mechanical stirring: one that makes use of an internal stirrer, acting as a complex scatterer inside the volume confined within the cavity, named in this paper as "Rotating Paddle", and another subclass that, conversely, uses changes in the wall(s) of a chamber to allow for mode stirring.

\section{a. Rotating Paddle(s)}

The rotating paddle (scatterer) is widely known as "mode stirrer" or just "stirrer". Other names that can be found in literature are: (mode) tuner, (mode) mixer, paddle wheel or fan.

The list of all the rotating paddles implemented around the world is extensive. We are unable to report on all the different shapes, sizes and strategies ever envisaged to create a successful stirrer. Some cases would really qualify for a modern art gallery, showing off the creativity and imagination of their designers. It is not exaggerated to state that almost every lab that has participated in the design of their own stirrer, has come with its own "recipe". In this subsection, mainly due to space constraints, we focus on the "Z-fold" stirrer (probably the most widespread stirrer design), two remarkable designs where the stirrer can change its shape very easily and a novel stirrer that will trigger nice memories of amusing times spent in merry-gorounds...

\section{i. The "Z-fold" stirrer}

The "Z-Fold" stirrer, sketched in Fig. 7, often found in many reverberating chambers was originally developed under a Cooperative Research and Development Agreement (CRADA) between Lindgren RF Enclosures (now ETS-Lindgren) and the Naval Surface Warfare Center, Dahlgren Division (NSWCDD). The agreement was entered into in March of 1997. The objective of the agreement was to design, fabricate and test a compact reverberation chamber that would operate down to a lower frequency limit of $80 \mathrm{MHz}$ as specified in International Electrotechnical Commission (IEC) 1000-4-3. The expected results were to obtain significant advancement in the application of reverberation chambers for conducting electromagnetic vulnerability testing of future commercial electronics and weapons systems. Under the CRADA, NSWCDD was responsible for providing design guidance for the reverberation chamber and providing Lindgren RF enclosures with a list of recommended components to construct a prototype reverberation chamber. Lindgren RF enclosures were responsible for overall chamber design, development of fabrication methods and construction 
of a prototype chamber. Lindgren RF enclosures were also responsible for installation of the required mechanical tuners and support equipment for the prototype chamber.

After multiple configurations of possible tuner arrangements were considered, the Z-fold design was selected as it provided the necessary perturbation of the RF fields, while at the same time providing a rigid structure that minimized the mechanical "damping" time required for the tuner to settle after being moved as well as minimizing the requirements of the drive system. It was also determined that two tuners oriented perpendicular to each other help minimize the amount of "unstirred energy".

The CRADA resulted in US Patent 6,686,818 B1 for "Reverberation Chamber Tuner and Shaft with Electromagnetic Radiation Leakage Device" which was filed on March 8, 2000 and issued on February 3, 2004. The inventors were listed as Joseph Weibler, Matthew Squire, Stan Zielinski, Dale Svetanoff, Michael Slocum and Michael O. Hatfield.

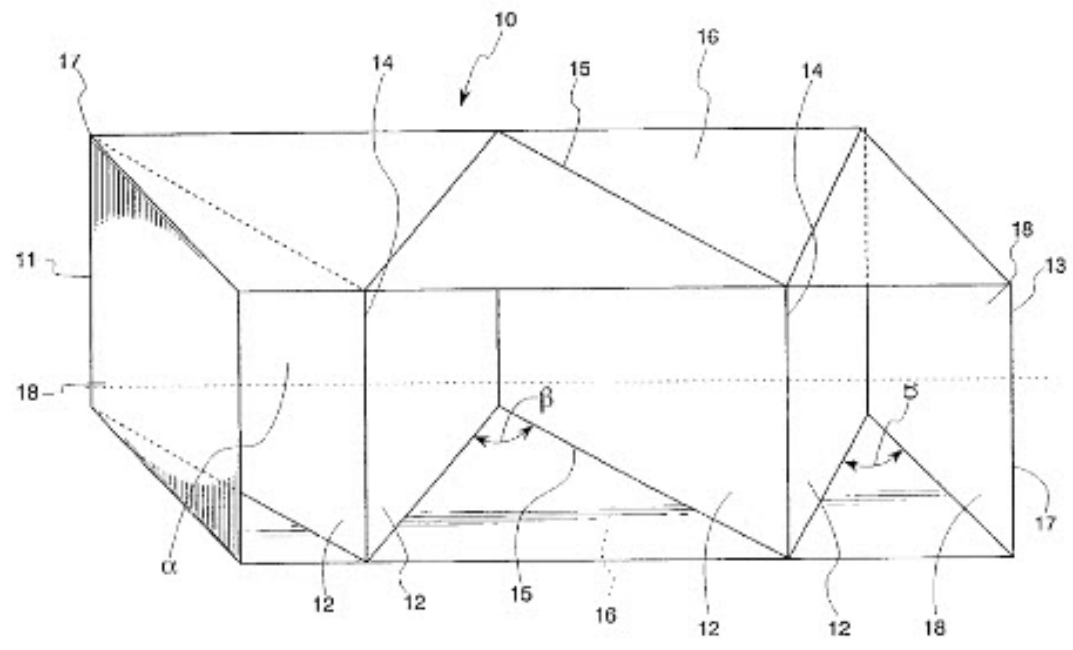

Fig. 7. The Z-fold stirrer. Figure taken from US Patent $6,686,818$ B1

The Z-fold stirrer has become the classical stirrer shape (e.g. it is the stirrer design present in the RC schematic diagrams of the standard [2]).

\section{ii. The "bent-plates" stirrer}

Figure 8 shows the "bent-plates" stirrer used in the RC facility at the University of York. The stirrer has eight identical panels on four arms. Each panel is set at a different angle to the vertical. The eight angles can be varied at will, rendering this particular stirrer reconfigurable and allowing for an optimization process. In each case the swept radius and total surface area are unchanged. A non-optimized design of a bent plate stirrer, using four bent plates with a fixed dihedral angle of approximately $133^{\circ}$ for each plate, was used in [9]. 


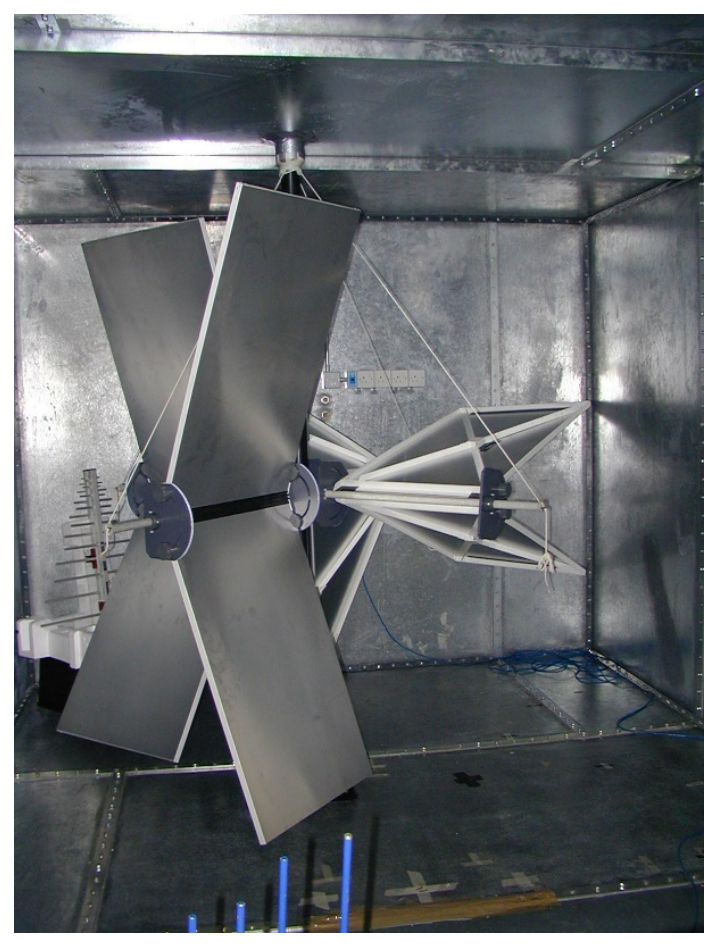

Fig. 8. The reconfigurable stirrer at the University of York.

The stirrer shown in Fig. 8 was developed from research at the University of York into stirrer optimization using a Genetic Algorithm (GA) [10]. The eight angles comprise the genotypes used in the GA. Such optimization process was performed against a specific metric devised at the same university [11]. Using time-domain transmission-line-matrix method in free space with plane wave illumination of the stirrer helps decreasing dramatically the computation time, typically from four full days when the stirrer is included inside the resonant cavity, to few minutes for the free space case as the energy leaves the problem space rapidly, unlike the reverberation chamber case. These simulation times apply to typical computational performance available back in 2004. A similar ratio would still apply today. The eventual metric used a number of elemental dipole sources randomly distributed on the surface of a sphere around the candidate stirrer. A set of observation points was also distributed on the spherical surface. The stirrer's performance is optimized by maximizing the average change in the direction of the Poynting vectors of the waves scattered by the stirrer compared to the waves scattered by a reflecting cube of the same volume of the stirrer.

The optimized stirrer was set up and its performance was measured and compared to a suboptimal stirrer using an aggregated measure of the difference between field uniformity measured in the chamber and the field uniformity limit set in the IEC 61000-4-21 Standard [2]. The optimized stirrer outperformed the non-optimal designs.

This work also showed that the optimal stirrer design can be scaled in size for use in different chambers. Currently, researchers at the University of York use the same design, i.e. the same set of eight angles, in two of their chambers which have dimensions $4.7 \mathrm{~m} \times 3 \mathrm{~m} \times 2.37 \mathrm{~m}$ and $0.8 \mathrm{~m} \times$ $0.7 \mathrm{~m} \times 0.6 \mathrm{~m}$. 


\section{iii. The irregular reconfigurable stirrer}

It has long been recognized that the size and shape of mode stirrers has a significant effect on the quality of the mode stirred field, in terms of its statistical uniformity and the closeness of probability distribution to that for ideal statistically random fields. However, apart from the basic requirement of the chamber needing to be sizeable compared to the wavelength, the question of "complexity" of the mode stirrer has been difficult to address. Even designs produced by optimization algorithms are highly non-intuitive and may depend on the scale of resolution applied. Some numerical optimization results and general design rules have been formulated, but specific and practical designs that enable efficient stirring near the nominal lowest useable frequency (LUF) (for instance, around three to five times the first resonant frequency of the cavity) for use in rectangular chambers with smooth walls, have remained elusive.

In the pre-2000 era, only few results on the effect of stirrer shape were known and full-wave 3D numerical simulation of realistic chambers at short wavelengths was barely feasible. Meanwhile, computational power has now reached a stage where such simulations have become feasible. Nevertheless, simulation results obtained from the staircase approximation for modelled stirrers, which arises when modelling surfaces that are inclined at an angle with respect to a rectangular simulation grid, may suggest a deceptively good stirring performance, because of the effect of jagged edges on diffusion, diffraction, and the average mode density [12]. 


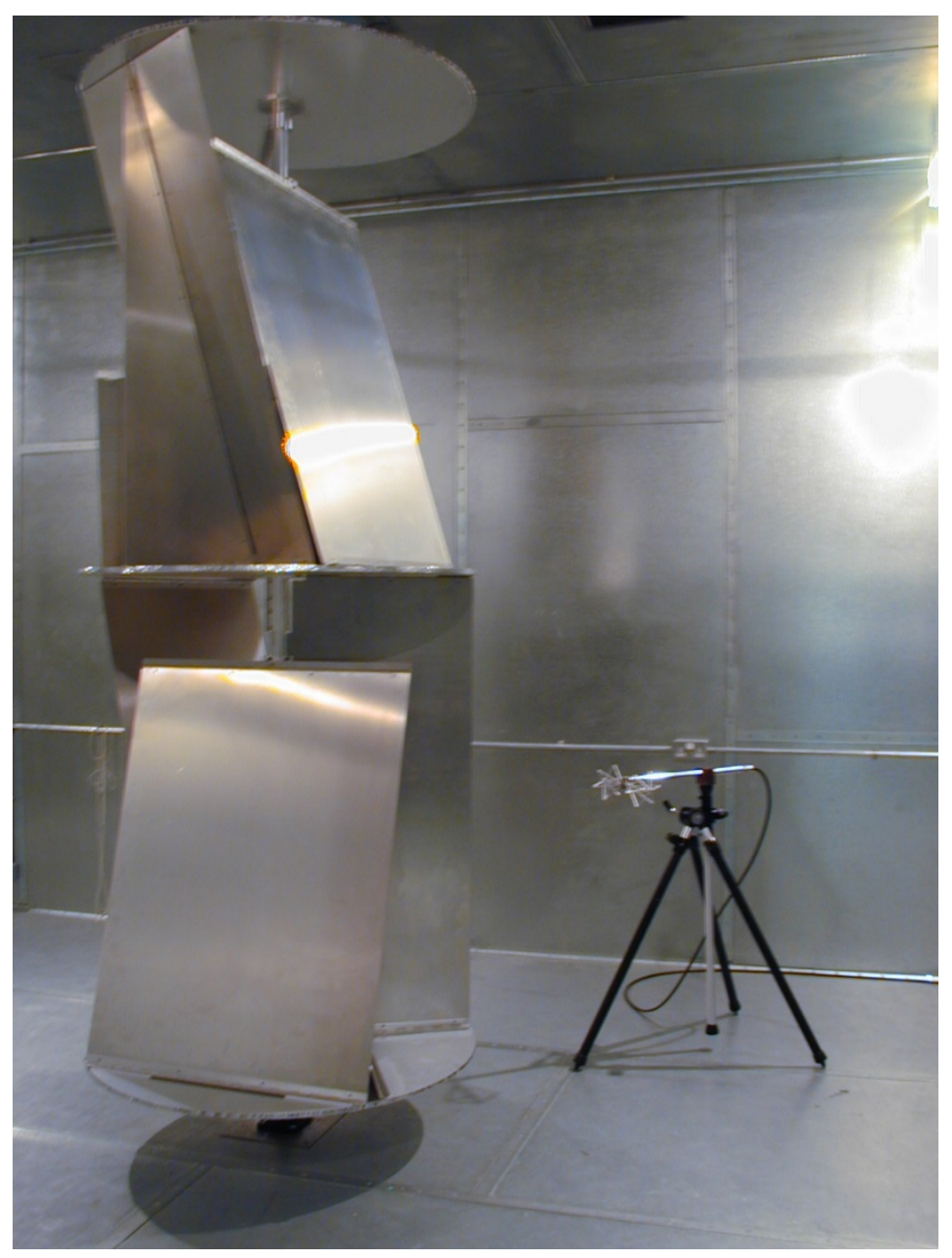

Fig. 9. Irregular reconfigurable stirrer in the RC at NPL, based on detachable aluminum stir blades and hinges that are attached to metal honeycomb disks using Velcro ${ }^{\circledR}$. Photo extracted from [13].

Therefore, a design for a paddle wheel in the rectangular chamber at NPL in Teddington, UK, commissioned in 2001 was implemented that enabled easy re-assembly and reconfiguration of its blades. To this end, the stirrer blades were attached with Velcro@ strips to three disk platforms, enabling their easy removal and reorientation (Fig. 9). This design was chosen to enable easy modification during experimentation and reconfiguration as further insights and results into stirrer design would become available [14]. Some limited experimentation during the validation phase of the chamber according to [2] showed that, when the largest blade had an inclination between $30^{\circ}$ and $45^{\circ}$ with respect to the vertical axis, this enabled the LUF of the empty chamber to be lowered from $175 \mathrm{MHz}$ to about $165 \mathrm{MHz}$, compared to a vertical blade (angle of $0^{\circ}$ ). However, since violation of the IEC limit only occurred at a few isolated frequencies, it remains uncertain whether any modest lowering of this kind could have equally been achieved using a strategically chosen frequency list, based on a different start frequency. More extensive empirical optimization of different configurations would require substantial additional effort, perhaps also including engineering intuition. 
Conversely, the use of reconfigurable blades with easy attachment and detachment can also be used in more fundamental studies of canonical configurations as in [15] to understand stirrer performance.

While optimization of the stirrer's geometry has been a major focus point, the role of the path traced by the stirrer is equally important. This aspect has been recently investigated in [16].

iv. The carrousel stirrer

Considering the case of rotating paddles, the stirring efficiency depends on paddles size, rotating volume, and eccentricity [17], [18], and an optimization leads to better performance [10]. Typically, the stirring efficiency is enhanced by enlarging the paddle dimensions as much as possible, even though this could lead to a progressive reduction of the WV available for testing. On the other hand, it has also been demonstrated that it is the peripheral part of the stirrer blades that gives the main contribution in generating uncorrelated samples [18]. In that way, stirrer blades can be reduced in weight and construction complexity [19]. Merging these two conditions, a new stirring system can be achieved by nesting the WV within the stirrer rotating volume in order to increase as much as possible the paddle rotating diameter and at the same time to increase the WV as much as possible.

Let us consider a metallic rotating blade whose width is just smaller than the width of the chamber and the same for its height. Let us remove the inner part of the blade leaving only two peripheral strips whose widths are fundamental for the reverberation chamber (RC) performance. In that way, the free volume within the two strips can be used to locate the WV. Of course, other strips can be added to improve the chamber performance, obtaining a sort of "carrousel" that rotates around the WV, as sketched in Fig. 10. Compared to a traditional Z-fold stirrer in the same chamber [20], this new system provides better field uniformity and more uncorrelated angular positions. We achieve 70 uncorrelated positions at a frequency equal to six times the first resonant frequency with only 2 strips $(25 \mathrm{~cm}$ wide) and 130 positions with the 8 strips of Fig. 10, whereas the traditional Z-fold stirrer (1.2 $\mathrm{m}$ of rotating diameter) yields only 40 positions at the same frequency [21]. Figure 11 shows the field uniformity computed according to [2] for the new system equipped with 2-8 blades; at the frequency of study, the traditional Z-fold stirrer returned $2 \mathrm{~dB}$ for the averaged uniformity. A mechanic drawback of this new system could be a little bit more complicated engine to move the carrousel w.r.t. a single traditional paddle. 


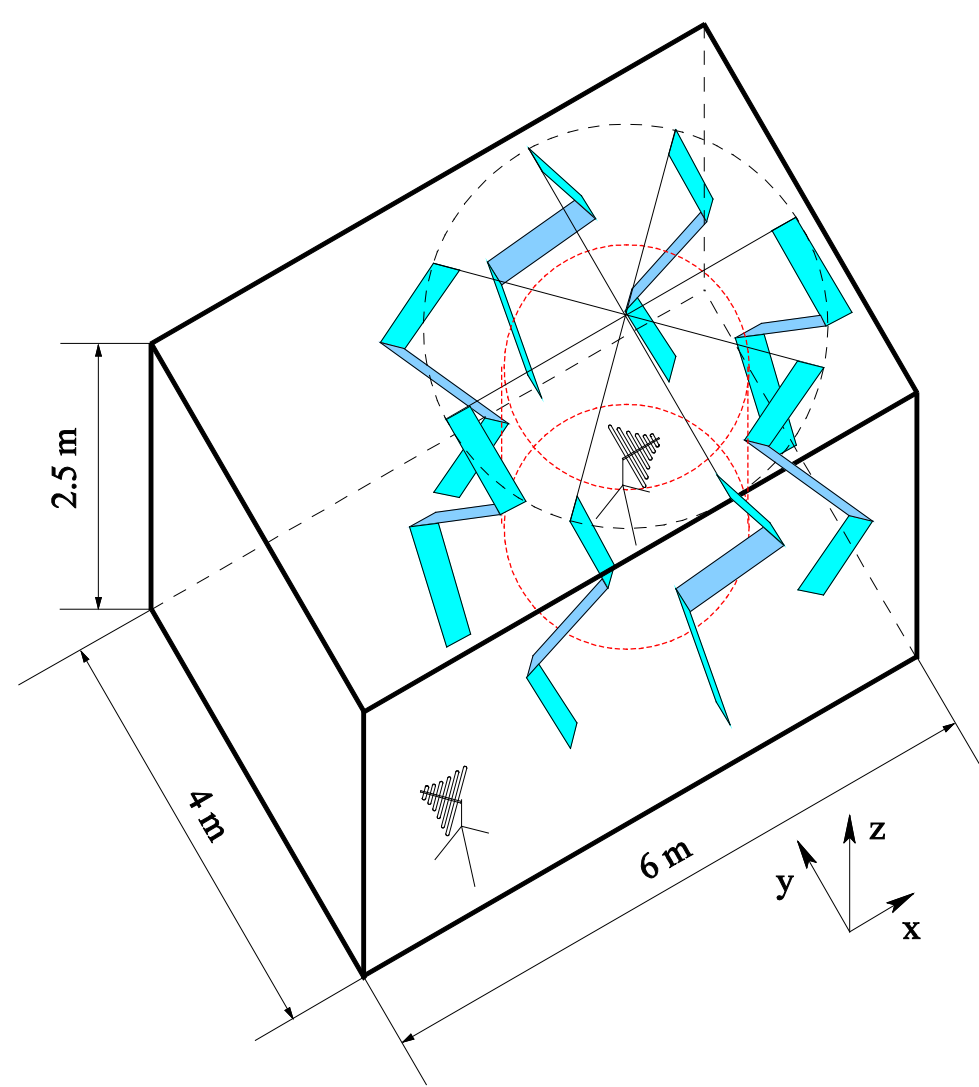

Fig. 10. Geometry of a RC equipped by an eight-strip carousel stirrer. The WV (red dashed line) has a diameter of $2.3 \mathrm{~m}$ and an height equal to $1.5 \mathrm{~m}$, in order to maintain the field computation points at a distance of $50 \mathrm{~cm}$ from the chamber floor and ceiling.
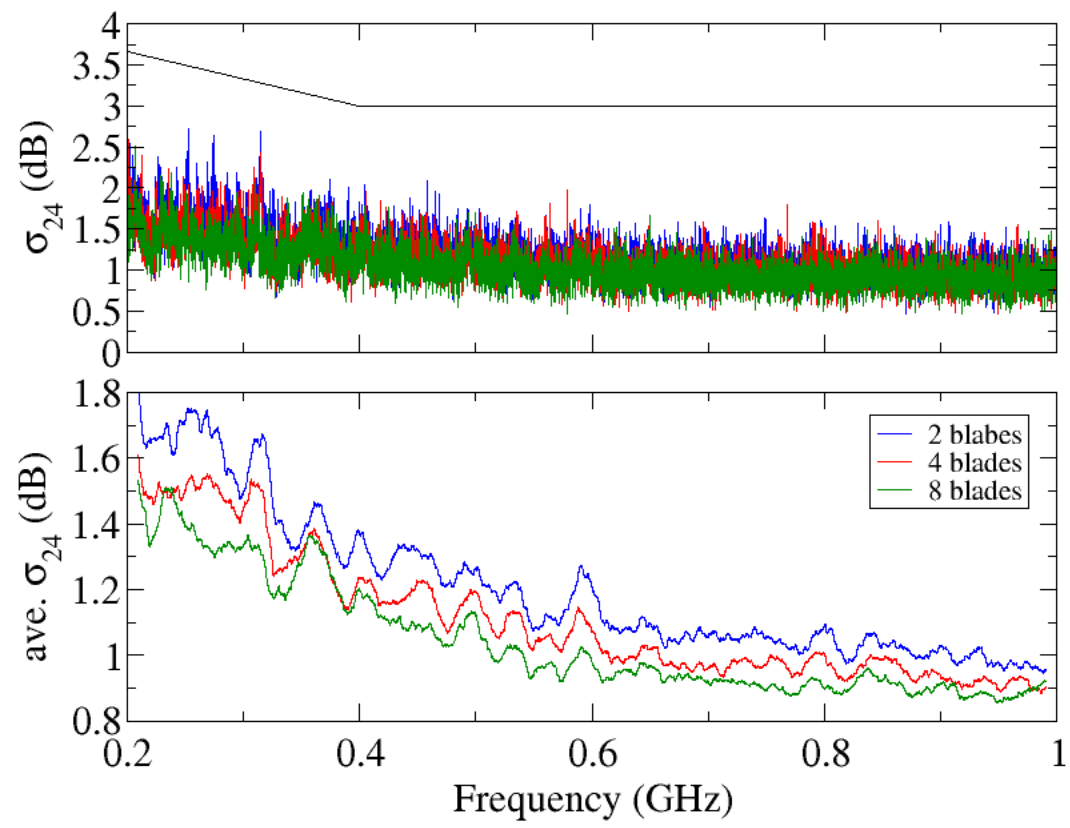
Fig. 11. Field uniformity as a function of the carousel strip number compared to the IEC 61000-4-21 limit: raw data (upper) and averaged data (lower).

\section{b. Wall stirring techniques}

Instead of using a chamber with an interior stirrer, a different example of mechanical mode stirring is represented by the RCs that, generally due to a particular movement of one or several of their walls, change the modal structure at every stir state by performing "wall-stirring", a second sub-class of mechanical stirring. Some early, mostly theoretical, work was introduced in [22], where a chamber with a moving wall showed good field uniformity characteristics.

Experiments using this simple wall movement were never performed in practice, but many other wall stirring techniques have been developed and practically realized in the past years.

\section{i. VIRC}

The EMC and acoustic laboratories of Thales Nederland (then Hollandse Signaalapparaten B.V.) were located in the same building. The acoustic laboratory featured a reverberation room ${ }^{1}$. An acoustic reverberation room has non-parallel walls, ceiling not parallel to the floor and at most two walls are placed at a normal angle with respect to an adjacent wall. Moreover, every wall area-to-wall area ratio is different. In [23] the suggestion was made to use an acoustic reverberation room as an electromagnetic reverberation chamber by covering the wall with metal. A first chamber was built sewing metalized cloth to a "tent" of approximately $2 \times 1.8 \times$ $1.5 \mathrm{~m}$ as shown in Fig. 12. Although the metal cloth created a complex shaped curvature which intuitively should have succeeded in diffusing the scattered electromagnetic field, this did not work out as expected. What works for acoustics, described by scalar fields with relatively slow waves (slow field build-up times) does not work (practically) for electromagnetics, described by vector fields with faster waves (fast field build-up times).

\footnotetext{
${ }^{1}$ It is curious to notice that within the EMC terminology, reverberation chambers are "chambers", while within the acoustic terminology these are "rooms".
} 


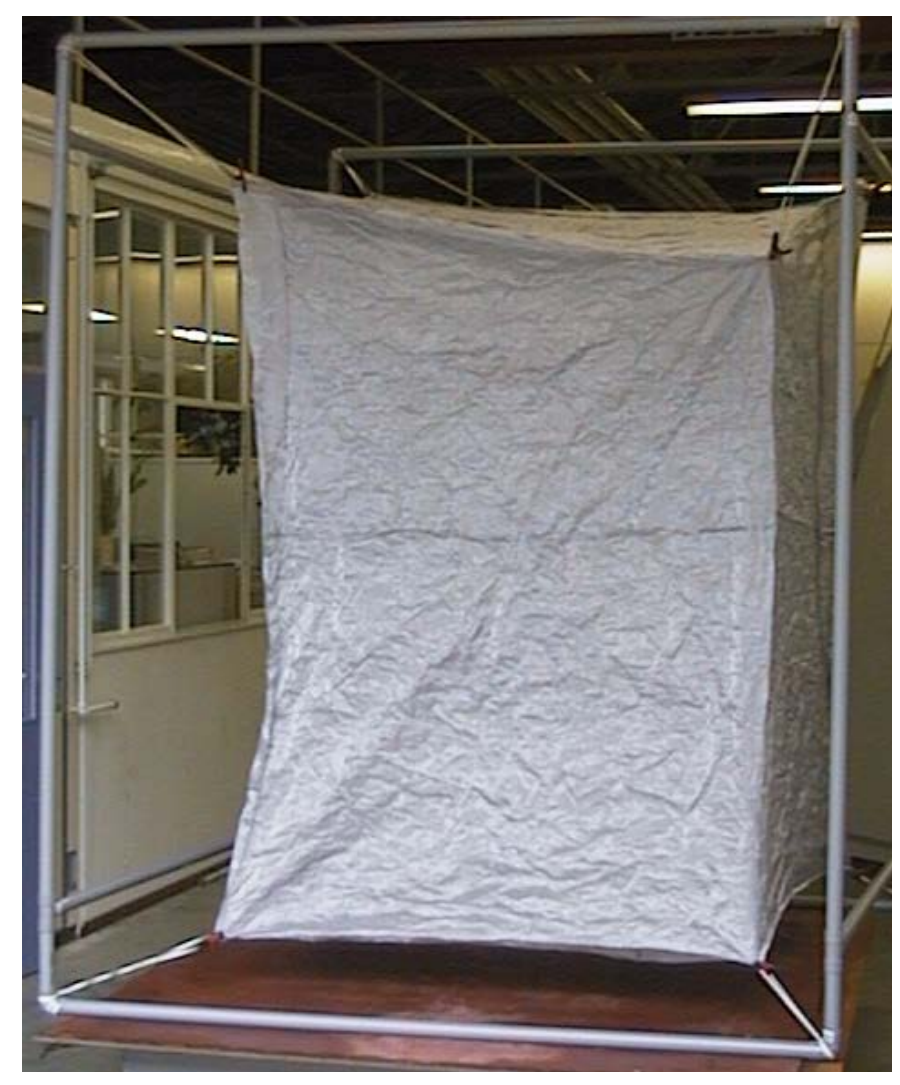

Fig. 12. The Vibrating Intrinsic Reverberation Chamber hanging on strings

But by changing the angles of the wall-floor-ceiling of the tent (i.e. by shaking the tent) resulted indeed in the desired statistically uniform electromagnetic field. By this stirring mechanism the field proved to be randomly polarized, spatially uniform and isotropic. Such a chamber is called the Vibrating Intrinsic Reverberation Chamber (VIRC) [24], [25], [26]. In essence, the VIRC is a RC where the walls are made of flexible conducting material and there is no interior stirrer. By moving one or more ridges or one or more walls the modal behavior of the field inside the chamber is changed, and thus the resonance frequencies are changed.

This stirring technique generates a frequency shift in its modal structure that is much larger compared to what is possible with a classic mode stirrer. This implies that the frequency range of operation of the VIRC, is extended to lower frequencies compared to (traditional) reverberation chambers with equal dimensions.

Quite often, the VIRC is compared to a RC with a moving wall. A moving wall changes the modal structure, indeed, but its influence at relatively low frequencies is mild. The key element is the translation of multiple walls of the VIRC which enhances reflection of fields in all directions. Therefore, some VIRC users claim that this particular chamber can be used at much lower frequencies as a comparable sized fixed-wall RCs.

A considerable number of VIRCs are in use by research institutes and industry, exploiting one of its main advantages: the possibility for in-situ testing. Instead of having to bring the Equipment 
Under Test (EUT) to the lab, the VIRC can be taken to, and installed around, the EUT. The VIRC has been and is being used to test various radar systems, parts of planes, and complete satellite systems, as shown in Fig. 13.

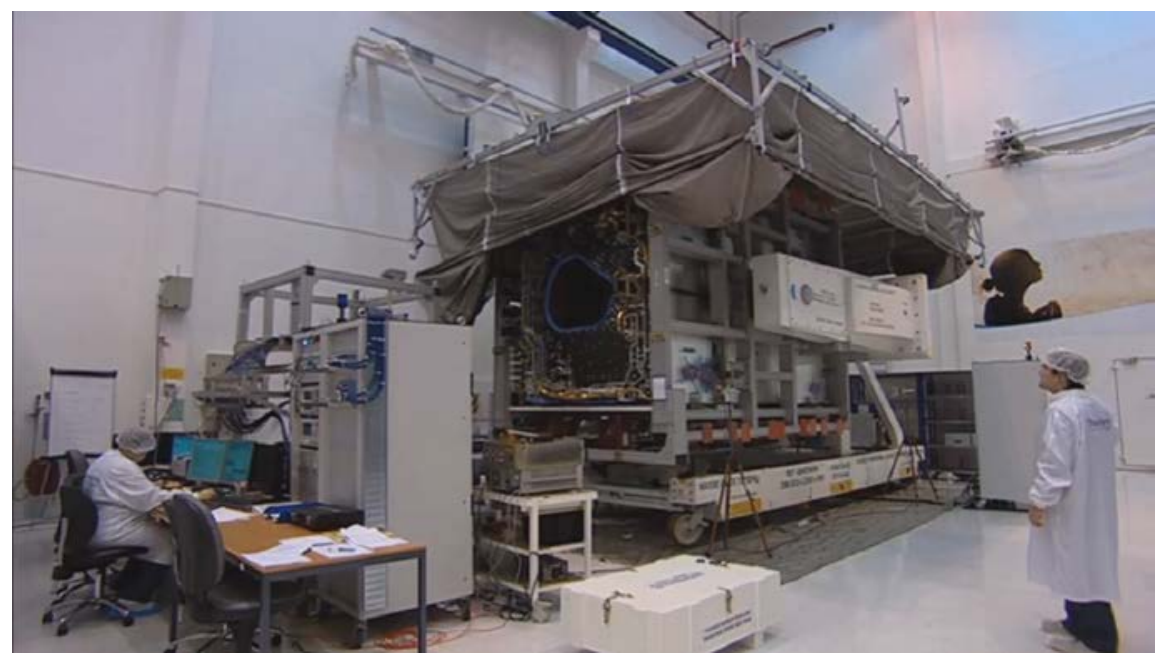

Fig. 13. Using the VIRC for the "sniff and spray test" for satellites. The "sniff and spray tests" are leakage tests on the several thousands of waveguide junctions in a high-tech communication satellite. This test takes normally four weeks with conventional test setups, reduced to few days when the VIRC is used.

\section{ii. Stadium Chamber}

Typical reverberation chambers are based on a rectangular cavity, for practical reasons. Employing more complex-shaped surfaces, in particular including curvature, may help in improving performance, particularly at low frequencies. Not only do curved surfaces have diffusive or focusing properties, they also enable increasing the average mode density at relatively low frequencies, e.g. [12]. This may assist in decreasing the LUF.

Partially based on ideas from quantum chaos in so-called 2D ergodic billiards, an original design for an integrating sphere at NPL was converted into a 3D stadium shape by inserting a cylindrical section of variable height. For use at frequencies above $8 \mathrm{GHz}$, two aluminum hemispheres of nominal radius $35 \mathrm{~cm}$ were joined through a cylindrical section of height $17.5 \mathrm{~cm}$. Smaller convex spherical caps with opposite (negative) curvature were added onto the inner spherical surface, which provide dispersion (diffusion) of waves. Figure 14 shows the external and internal (lower hemisphere) views of the Stadium chamber at NPL. In combination with making the lower hemisphere rotatable using a belt drive mechanism, these produce a wall stirred "chaotic" cavity with curved corrugations. The cavity has been operated in mode-tuned and mode-stirred operation (to at least 120 revolutions per minute). 


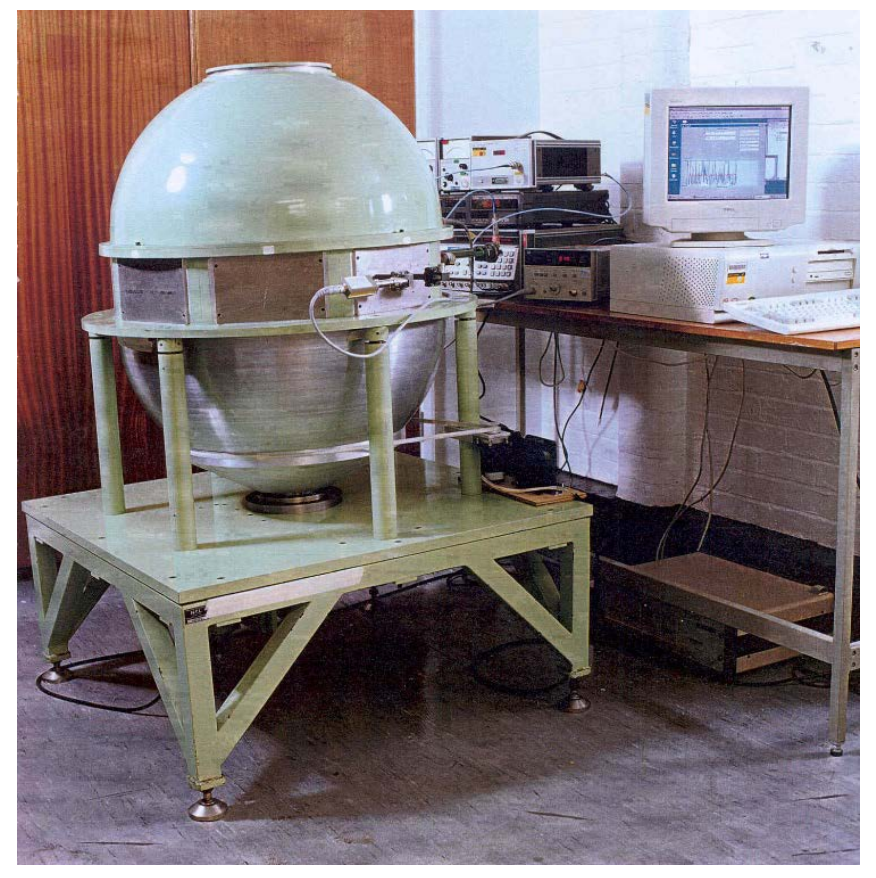

(a)

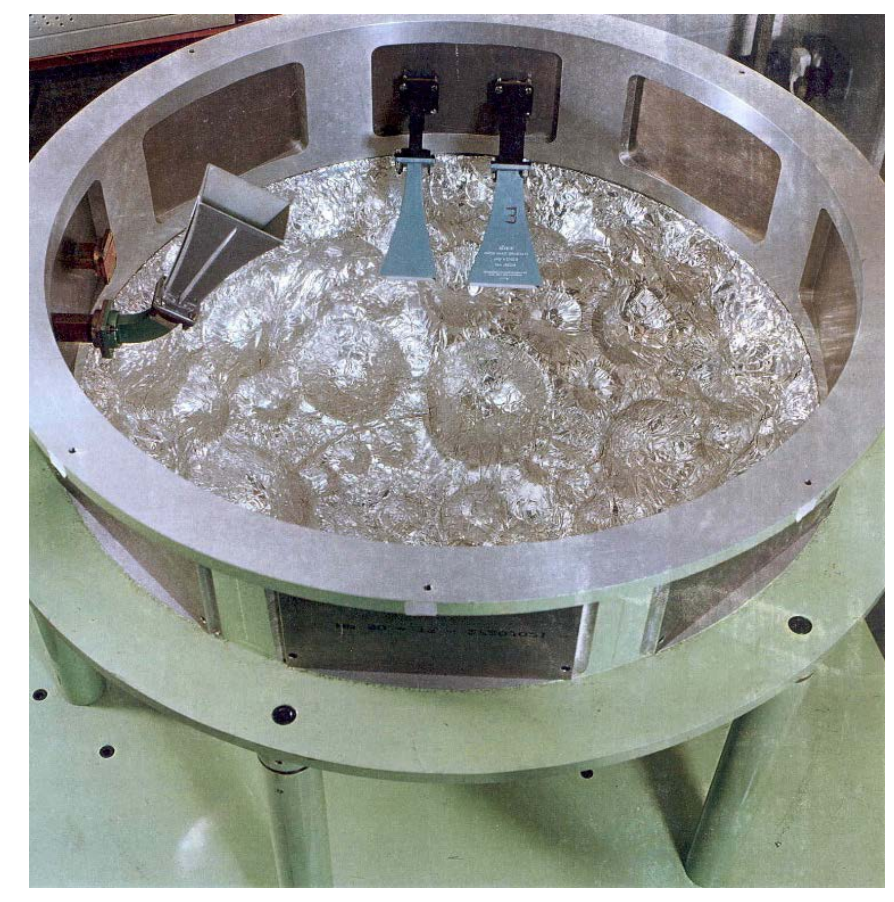

(b)

Fig. 14. (a) External and (b) internal (lower hemisphere) views of stadium RC at NPL, based on convex spherical cap diffusers of different sizes placed on the rotating inner wall of the cavity. Photos extracted from [27].

This stirring mechanism is highly efficient because there is no unstirred energy, as all internal reflections 'hit' a stirrer of maximum size that cannot be 'missed'. This is in contrast to e.g. an 
interior rotating paddle wheel, for which increasing its size implies a consequential reduction of the working volume. Secondly, unlike wall stirring designs based on flexible walls (EM 'tents'), the inner volume, surface area, local curvature of the walls and edge length are all preserved during stirring. This ensures a constant average mode density during stirring. As a result, all states of the cavity are statistically equivalent and form a so-called ensemble, as in the case of a rectangular chamber with rotating central stirrer. This constancy facilitates the calculation of statistical field properties.

Its performance in terms of field isotropy and field homogeneity levels has been detailed in [27], [28], [29], [30].

It is worth noting that a design of what amounts in effect to "partial wall stirring" - although not exploiting curvature but based on low-profile rotating vanes in close proximity to the cavity walls that perturb the boundary field - was already proposed and implemented early on, in [6] and [7], [31].

\section{iii. Oscillating stirrer (Quasi-wall)}

This particular technique features a metallic stirrer which is still envisaged and designed as an interior moving scatterer analogous to the traditional rotating stirrers but contrasting to those in the fact that it does not rotate. Figure 15 shows an example of such stirrer present in the RC facility of the Eindhoven University of Technology (TU/e), The Netherlands. The oscillating stirrer fashions an irregular (i.e. non flat) wall inside the chamber with several hinges. Its oscillating movement, produced by a linear motor, resembles an expanding-contracting bellow. The stirrer allows for mode-stirred or mode-tuned regimes.

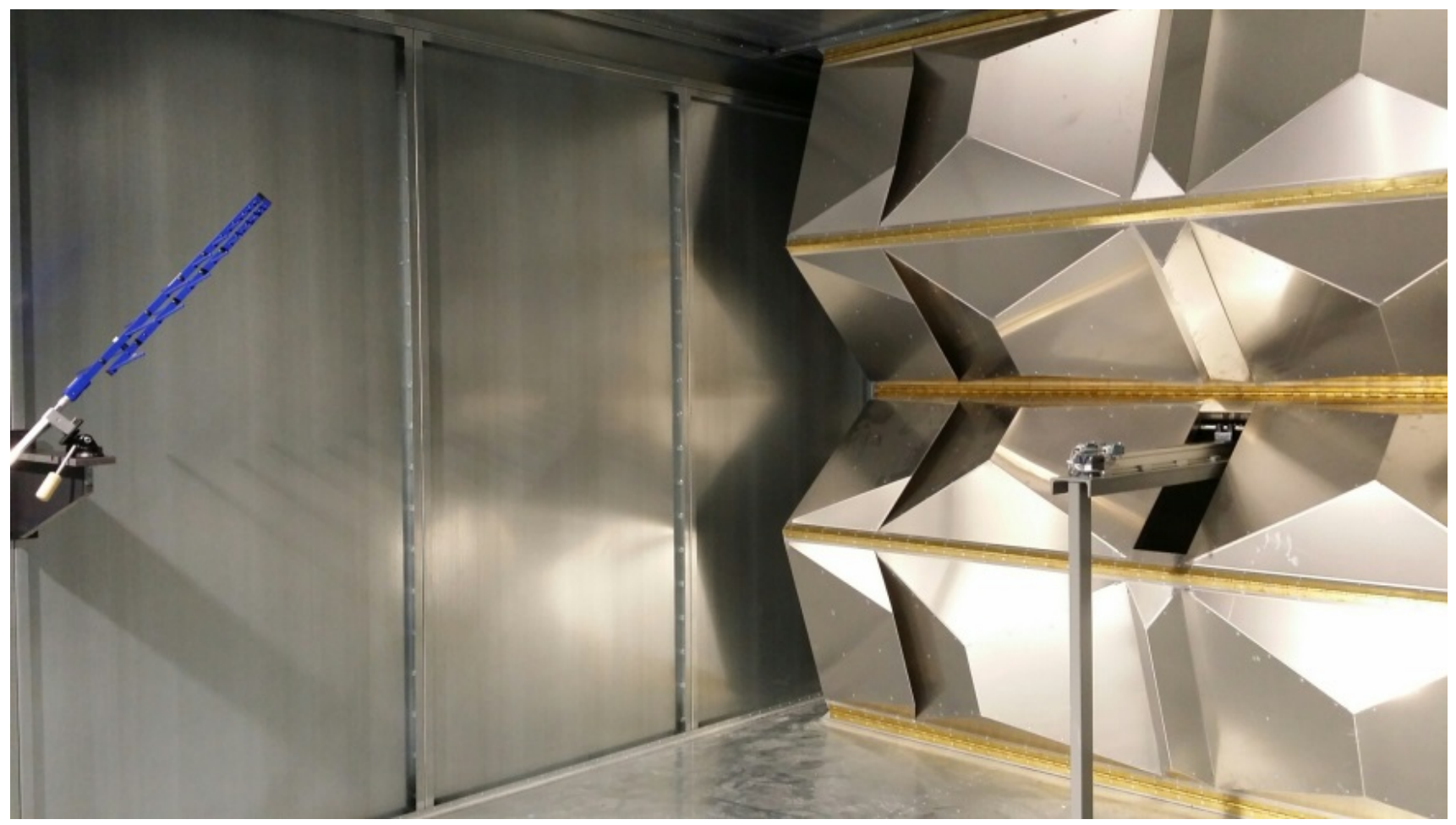


Fig. 15. The Oscillating Stirrer at the RC facility of the Eindhoven University of Technology. Five hinges allow for an expanding-contracting bellow-like movement of the stirrer, excited by a linear motor.

Though the oscillating stirrer is technically not a proper wall of the cavity, it can arguably be considered as a quasi-wall, because the space between the stirrer and the (actual) wall of the chamber remains yet relatively small. In this sense, it can be compared to the rotating vanes of [6], which have been, indeed, named "partial wall stirring".

One of the main advantages of this technique is the increased working-to-total volume ratio, compared to the traditional stirrers, by occupying more efficiently the room within the cavity. It follows naturally the rectangular shape featured by most cavities, which is significantly convenient from the practical point of view. The "space efficiency" of this RC is comparable to other wall-stirring techniques, like the VIRC or the stadium chamber.

Due to the fact that the oscillating stirrer is implemented in "normal" rectangular cavities, it is practical for a widespread use in EMC testing, while assuring a highly shielded environment. The stirrer can be moved at will, allowing repeatability of a stirring cycle, whenever this aspect is of importance and shares the same capabilities of traditional RCs used in immunity testing.

Field uniformity inside the RC of TU/e $(4.05 \mathrm{~m} \times 5.7 \mathrm{~m} \times 3.15 \mathrm{~m})$ was measured according to [2] and shown in Fig. 16. The RC facility of Delta Engineering in Denmark also uses the oscillating stirrer and results for this chamber are reported in [32].

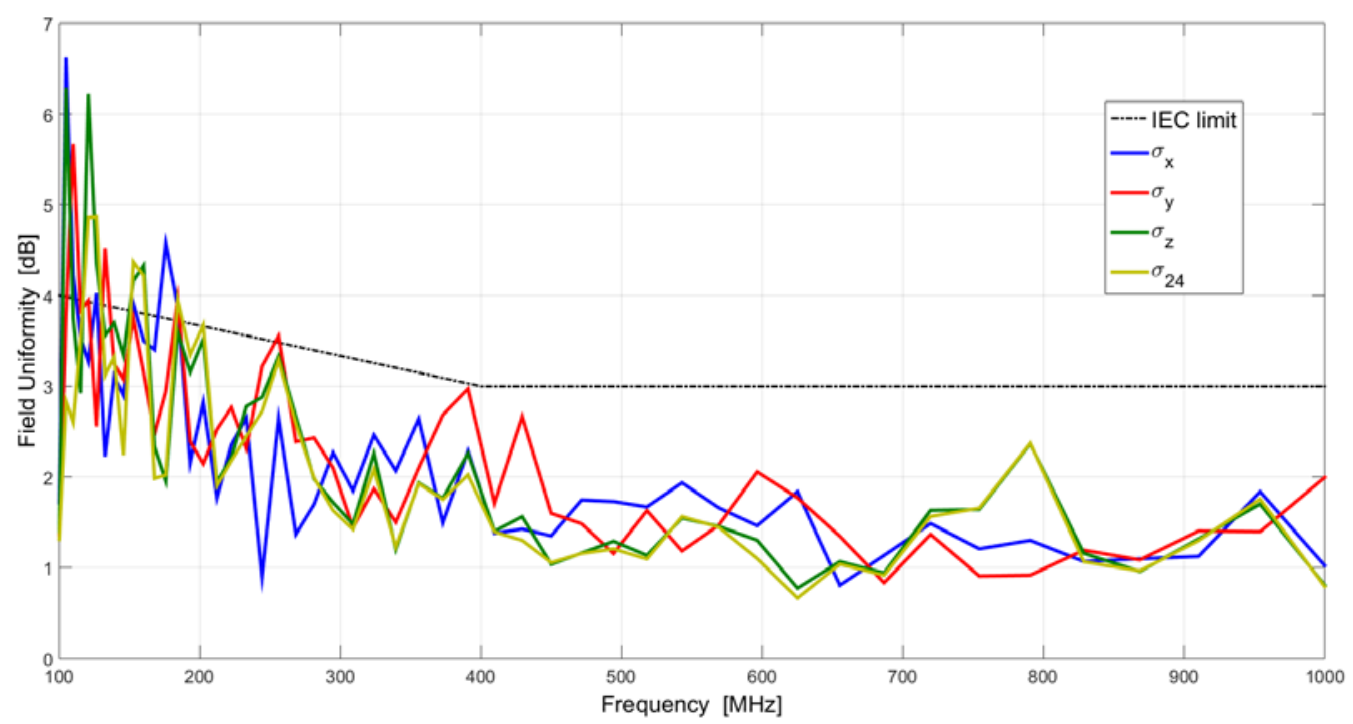

Fig. 16. Field uniformity measurements, according to [2], for the RC at TU/e (4.05m x 5.7m x 3.15m) featuring an oscillating stirrer. The quantities $\sigma_{x}, \sigma_{y}, \sigma_{z}$ and $\sigma_{24}$ are defined in [2] and represent the different metric for field uniformity. 


\section{Electronic Mode Stirring}

We denominate "electronic" mode stirring to those techniques in which the time-changing boundary conditions are achieved by electronic means. Opposed to mechanical stirring, they do not comprise the movement of any part of the RC. Electronic stirring techniques cover a broad spectrum of strategies, from shifting the frequency of excitation, to using multiple sources. Some of these techniques can arguably be considered "mechanical" like, for instance, the source position stirring, but the evolution of this particular technique has landed closer to electronic stirring than to mechanical stirring.

Electronic stirring techniques attempt to cope with some drawbacks of mechanical stirring, for instance (though not applicable to all mechanical stirring techniques):

- the volume that internal rotating paddles occupy is relatively important compared to the size of the chamber,

- the time taken to move the stirrer or the wall(s), from one stir state to the next one,

- the time needed to stabilize the stirrer once it reached the desired position. Fast-stabilizing stirrers are also more rigid and therefore, more costly and heavy,

- the mechanical motion involved requires powerful motor(s), shafts, gears, etc. and therefore maintenance.

However, electronic stirring seems not to benefit from the same popularity and widespread use as mechanical stirring. It is to be understood deeply the reasons why...

\section{a. Frequency stirring}

Assuming the ergodic hypothesis to be valid, then convergent statistical properties of a wellfunctioning RC will remain invariant if the changing factor becomes the frequency of excitation rather than, for instance, some geometrical factors. If instead of exciting the chamber with a continuous-wave (CW) signal, which is narrow band and thus needs mode overlap, a frequencymodulated signal with a large band is used, then the effect of mode stirring could be achieved. This is basically the principle of frequency mode-stirring.

Early work involved using wide band Gaussian noise to excite a chamber over octave bandwidths [33]. Frequency stirring using narrow bandwidths of white Gaussian noise were first reported in [34] in which controlled bandwidths of white Gaussian noise were analyzed for use in conducting electromagnetic susceptibility testing. The process developed by Loughry used narrow band filters $(5,10,25$ and $50 \mathrm{MHz})$ to generate white Gaussian noise with bandwidths of 10, 20, 50 and $100 \mathrm{MHz}$ respectively as shown in Figs. 17 and 18. 


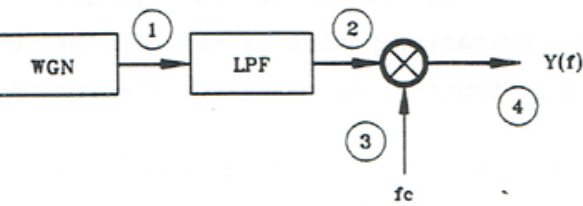

(1)

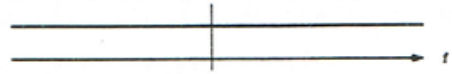

(3)

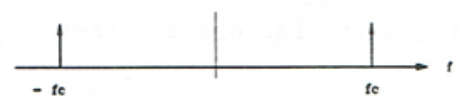

(2)

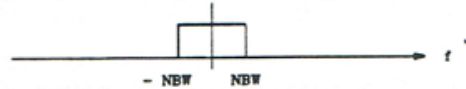

(4)

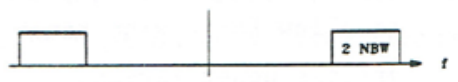

Fig. 17. Frequency Stir Spectrum. Picture taken from [34].

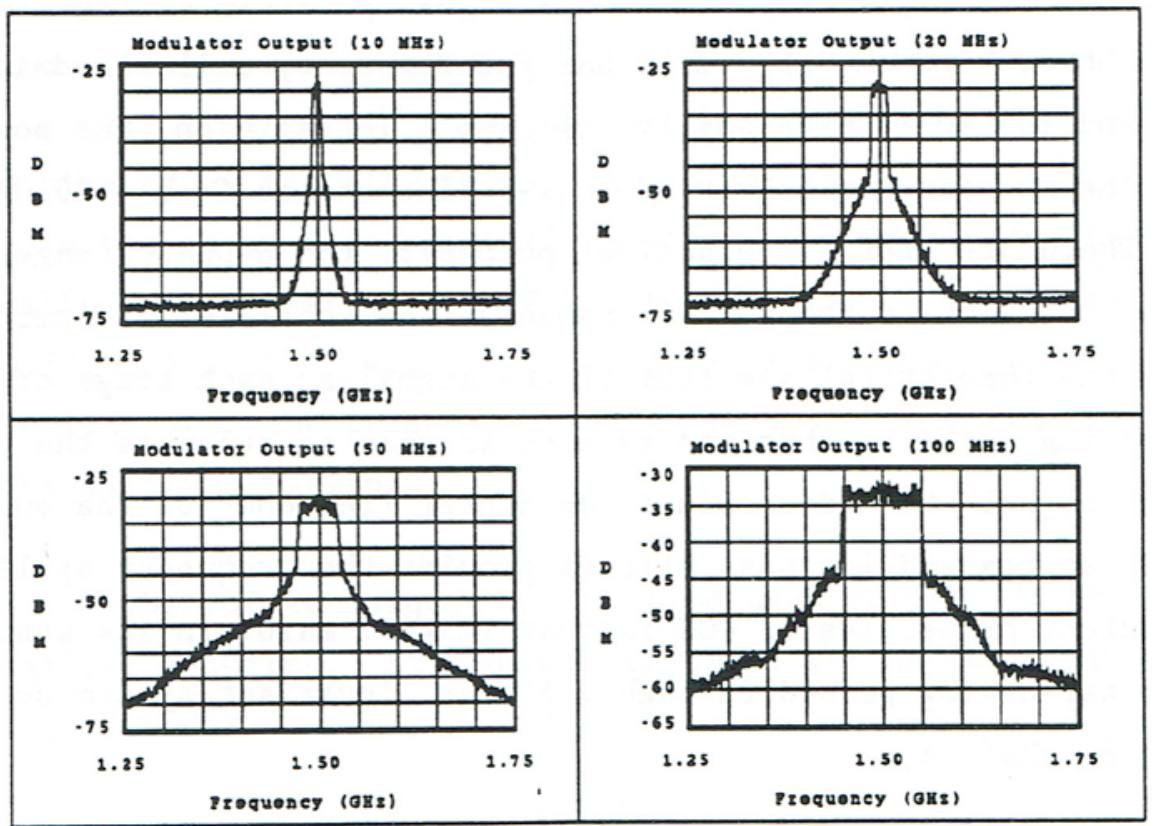

Fig. 18. Output of up converters for a center frequency of $1.5 \mathrm{GHz}$ for four agility bandwidths. Picture taken from [34].

Practical measurements using a band-limited white Gaussian noise (BLWGN) excitation are reported in [35]. The proposed measurement system in this report is to perform susceptibility tests. One of the main advantages that this type of operation claims is that of achieved real-time field uniformity, and not waiting for averaging over one stirrer rotation (or a full stirring cycle). It is declared that the test time could be reduced by a factor of 200 compared to mechanical stirring. The report also highlighted how the response bandwidth of the device under test must be taken into consideration when selecting the bandwidth of excitation.

\section{b. Random multiple-antenna stirring}


The low-frequency regime of RCs is characterized by a limited number of resonant modes overlapping [36], typically resulting in field distributions across the $\mathrm{RC}$ to be dominated by the most resonant mode, without having underlying ones providing comparable contributions that would ensure a more uniform field distribution.

A solution to this imbalance among modes is to excite RCs through multiple antennas. The idea in this case is to provide patterns of excitation signals that would enable exciting modes independently, thus also those not strongly resonant. In other words, to equalize the average contribution of each available mode at the frequency of operation of a RC.

Originally considered in [37] for the case of random independent excitation signals, the performance of multi-antenna excitations was shown to be rather disappointing, as the resulting stirring did not deliver any measurable improvement with respect to metrics such as field uniformity.

Yet multi-antenna excitation can be put to good work, using an alternative approach, namely the multi-antenna stirring technique, or MAS, introduced in [38]. Consider a RC excited by a set of antennas, instead of just only one, as it is usual. Each antenna is fed by a time-harmonic signal of a certain amplitude. The different combinations of signals exciting the different sources give rise to different stir states, analogously to different positions of a stirrer, for instance. Intuition would suggest that good mode-stirring would be possible should these excitation signals be random and statistically independent enough, as in [37]. Nevertheless, as it is proved in [38], the optimum mode-stirring is found when these excitation signals are partially correlated. This result is indeed surprising, especially if one does not read [38].

The explanation to this apparent challenge to intuition is not trivial, though. Let us just, for the sake of brevity, attempt a brief explanation. Readers can refer to [38] for a more detailed and rigorous explanation.

Consider the fact that the total electromagnetic field, under multi-antenna excitation, is a superposition of the field distribution from each singular antenna. So, if one could "equalize" at each receiving point within the WV the individual contribution of each modal structure (in this technique, by adjusting properly the amplitude of a certain excitation signal feeding a certain antenna), then field uniformity could, in principle, be achieved. Ideal spatial uniformity, as defined in [2], is achieved when the maximum field at a point within the WV does not depart much (in practice, does not depart more than $3 \mathrm{~dB}$ ) w.r.t. the maximum field at a different position and orientation within the WV. This measure of "does not depart much from" is mathematically translated into asking that the covariance matrix (covariance of the fields between different positions within the WV) is equal, as close as possible, to the identity matrix.

When the considerations explained above are implemented, the (surprising) result is that excitation signals must be partially correlated. This partial correlation depends on the transfer functions between every excitation antenna and the receiving point. 
This idea was tested in a RC with a volume of about $13 \mathrm{~m}^{3}$, where five conic monopole antennas were placed orthogonally to its vertical walls. The matrix of transfer functions was measured between the antenna input ports and the 24 scalar field samples required by [2]. The effectiveness of the MAS technique is apparent in Fig. 19, where the LUF associated to the mechanical stirrer of the RC, found around $500 \mathrm{MHz}$, is shifted at $100 \mathrm{MHz}$ by the MAS.

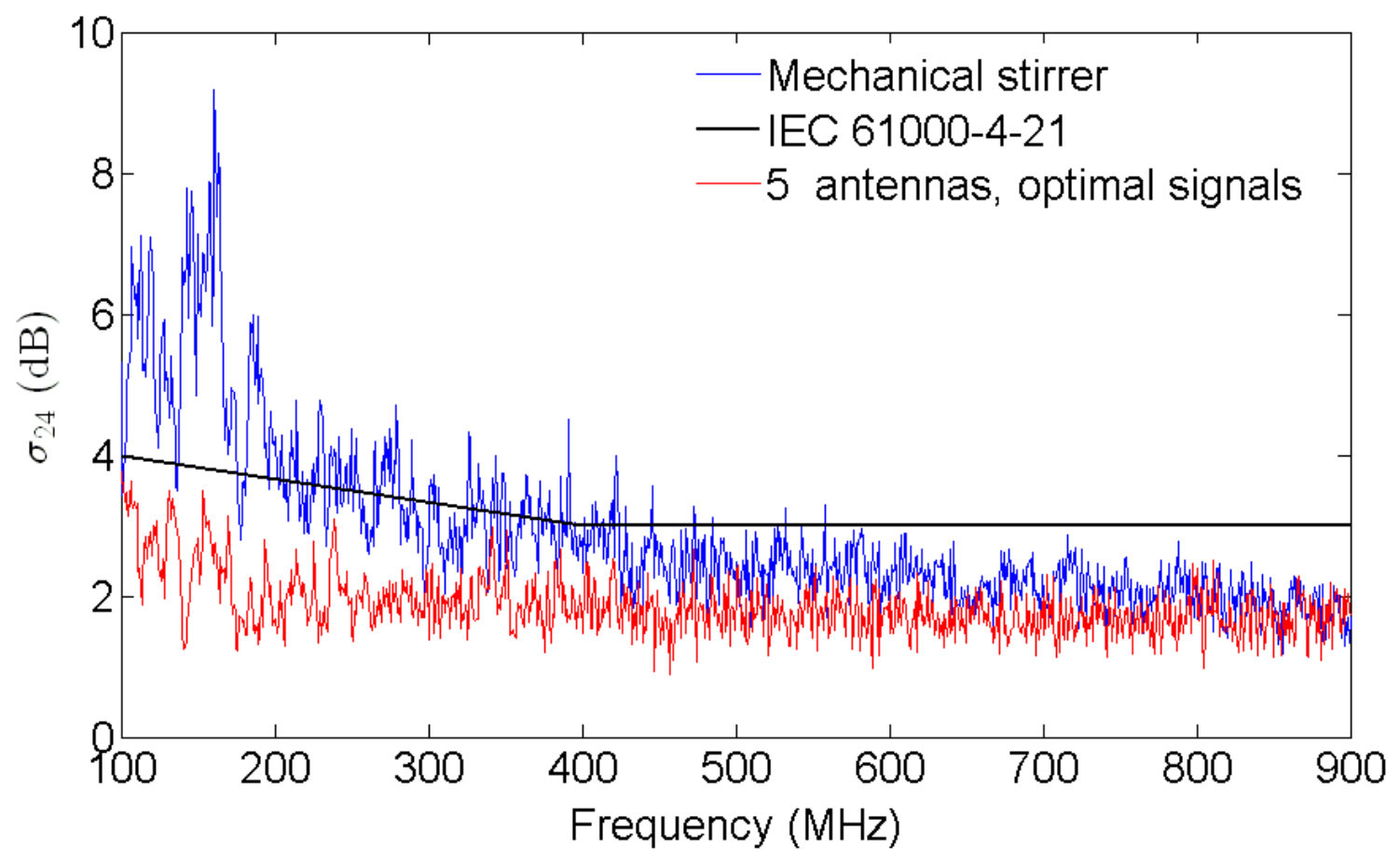

Fig. 19. Field uniformity results for a RC implementing the classical rotating stirrer and the comparison with the same chamber implementing the MAS technique.

This performance comes at the expense of multiple excitations, where relative amplitude and phase need to be controlled simultaneously and accurately, i.e., through multiple signal modulators. Moreover, the optimal procedure requires precise phase-dependent measurements that may be time consuming, and require phase-sensitive probes.

\section{c. Reactively-loaded antennas stirring}

The High Frequency Institute of the University of Dortmund has developed this kind of electronic mode stirring [39]. The basic idea is to attach many receiving antennas in the walls of the chamber. Each antenna is terminated by a variable reactive load. The effect of such configuration is that every antenna will receive a portion of the available electromagnetic energy inside the chamber and then re-radiate it back but with a varying phase. It is an electronic means to simulate the movement of a portion of the cavity wall. Varying the reactive load at each antenna position, will produce changing boundary conditions and thus mode stirring. First reported results in the gigahertz-range show promising future for this kind of stirring [39]. 


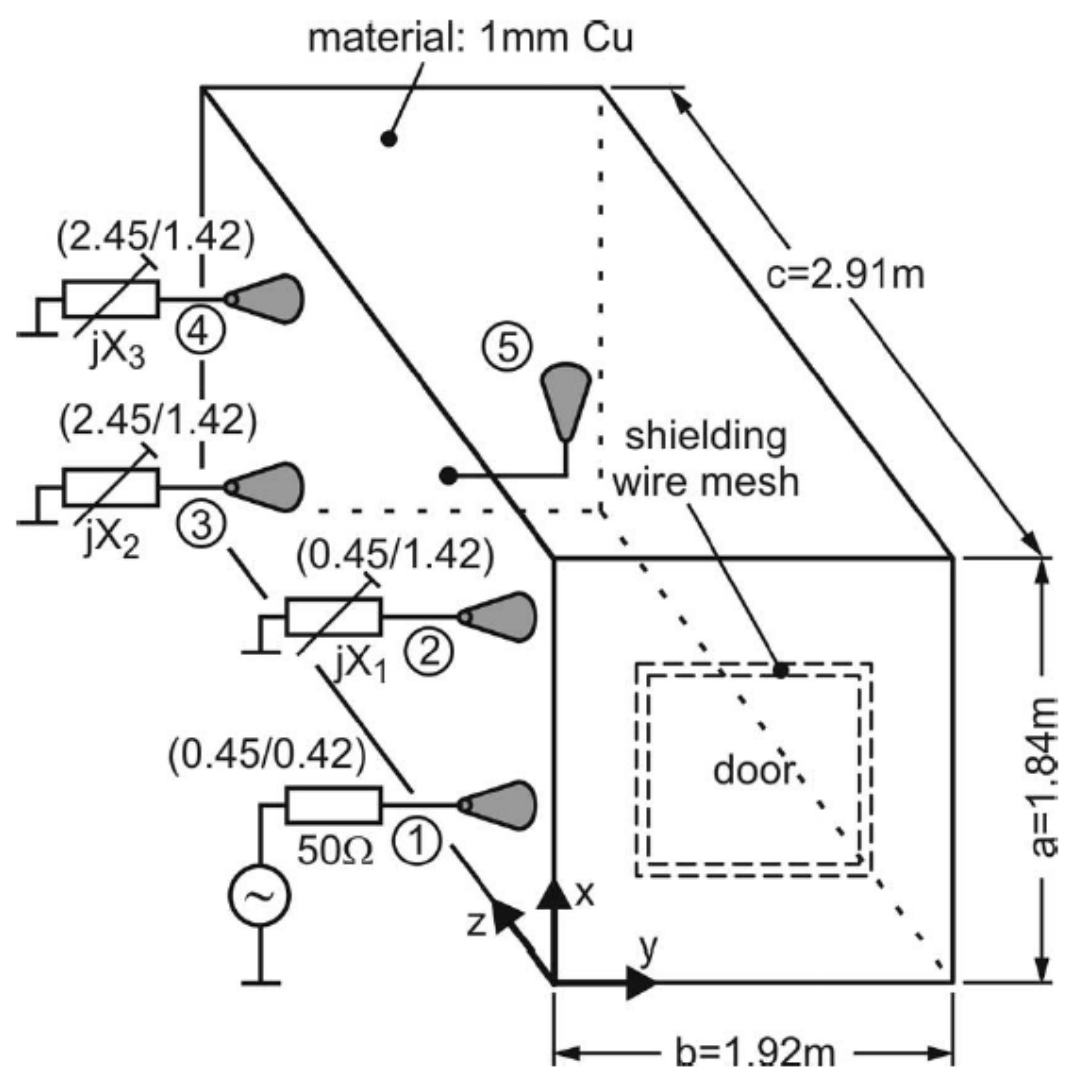

Fig. 20. Schematic diagram of a RC implementing the reactively-loaded antennas stirring. Antenna 1 is the transmitting antenna driven by a signal generator. Antennas 2-4 are loaded with variable reactances X1 - X3. Figure taken from [39].

A considerable advantage of this stirring technique is that the authors in [39] also provide a circuit model of such RC. This model is simple and allows for fast and accurate simulation of the field uniformity in the chamber.

\section{d. Source position stirring}

The idea of source stirred chamber was first introduced in 1992 [40]. In such a chamber, the source location inside the chamber is changed in order to produce mode-mixing. Indeed, as shown in [40], the electromagnetic fields inside a rectangular cavity are determined by the cavity modes and the source(s). For most mechanically-stirred chambers, the source is fixed whilst the cavity modes and their weighting factors are changed as the stirrer moves/rotates. For a source-stirred chamber instead, the cavity modes are fixed whilst the source location and the mode weighting factors are changed, thus the field at a point of interest is changed and a time-averaged uniform field could be obtained as in a mechanically-stirred chamber [22], [40], [41]. 
Since the introduction of the source-stirred chamber, there have been many studies on the field statistics inside such chambers as well as its realization. In [42], an experimental and theoretical study was conducted and the focus was on the statistical properties of the field from an operational point of view. It demonstrated the possibility to control the statistical properties of the field by source stirring. Their further study [43] has provided very encouraging results to demonstrate the real possibility to stir the field inside the chamber. Experimental and theoretical studies, e.g. [44] - [46] have further confirmed the validity of the source-stirred chamber.

The main advantage of a source-stirred chamber is the elimination of mechanical stirrers. Thus a larger WV could be obtained in such a chamber for a given site. It could also make the chamber more flexible and cost-effective. How to realize an effective source-stirred chamber has been a subject of research. For mechanically stirring the source could be complicated, alternative methods have been suggested. For example, instead of moving a single source to multiple locations, an antenna array could be mounted on the cavity walls to serve as the stirring source. An alternative to this approach could be arrays of antennas with different orientations and fed by an electronic switching network. As a consequence, the mechanical mode stirring could be replaced by electronic stirring without changing the source frequently. The main purpose is still to change the weighting coefficients of different cavity modes hence to stir the field inside the chamber.

Furthermore, there are some applications using the concept of source-stirred chamber outside the strict EMC domain. As an interesting example, Haier used the source-stirred concept to produce a new type of microwave ovens [47] in 2003. Unlike a conventional microwave oven, this new concept has no turntable inside and the field uniformity is achieved by changing the source.

\section{d. Switch Stirred Reverberation Chamber (SSRC)}

The principle of a SSRC, never experimented up to now [48], is essentially based on the modification of the internal electric field boundary conditions without moving any object or changing the internal geometry like is the case for mechanical stirrers.

It consists on installing several large metallic plates of different sizes at different given distances from the walls of the cavity such as schematically sketched in Fig. 21, where A, B, ..., G represent the different plates. These plates are then electrically connected to the respective walls of the cavity by electronically-controlled switches that will put each plate in contact or not with the cavity. The switches' location within each plate is a design parameter. 


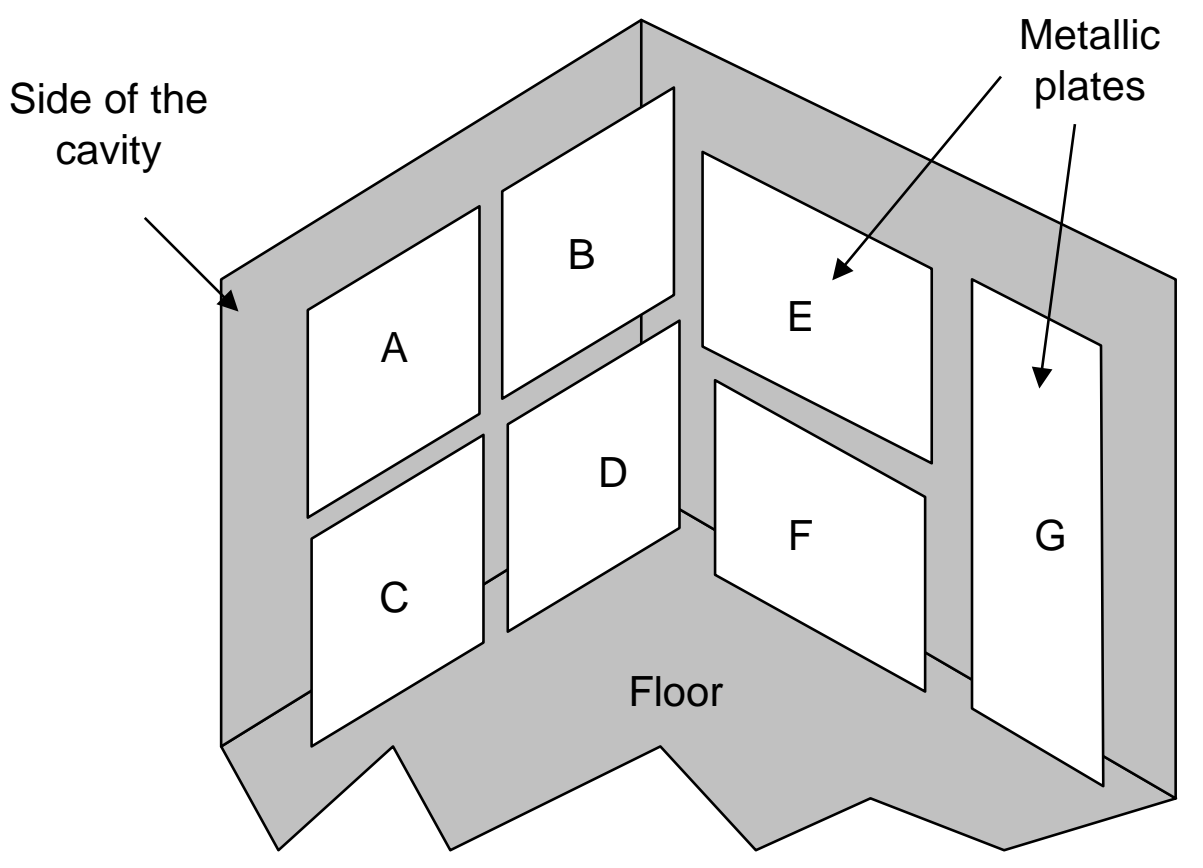

Fig. 21. Set of plates installed on the walls of a closed cavity

The boundary conditions can therefore be changed by electrically connecting and disconnecting individually each plate. In this way, to each combination of the switches in open or closed position will correspond a particular EM field distribution in the WV. The complete stirring cycle of the EM field will therefore consist in a more or less long sequence of states of the switches. For a SSRC consisting on $N$ plates, the total number of stir states is $2^{N}$.

The advantages of the SSRC are first of all related to the absence of a mechanical stirrer, which leads to a gain in terms of test volume, no stabilization time required between steps (instantaneous switching), and of course the cost savings on the stirrer, the electric motor and electrical accessories (power supply, sensors ...).

The advantages are also introduced by the use of independent electronically-controlled switches, which enable the end-user to program (and to reprogram easily) any type of successive combinations, any length of sequence (lower or equal than $2^{N}$ ) and any (independent) duration of each step depending on the stirring "performances" desired. This stirring technique could also considerably reduce the switching time between steps and offer very good repeatability.

Finally, such a stirring technique could be also very interesting in the case of very large structures such as metallic aircraft hangars where it is impossible to install a full-size mechanical stirrer. In this case, the plates could be constructed with aluminum foils to reduce their weight. 


\section{The maze of mode-stirring choices}

The main aim of this paper is not to compare performances between the different stirring techniques, but rather to list the different technologies proposed for mode-stirring in literature. Moreover, there exist a significant number of different metrics used to assess RC performance. A follow on paper overviewing the different performance indicators and figures of merit when assessing RC performance will follow in the next issue of this Magazine.

The significant number of the different available stirring choices should not mislead interested potential RC users into thinking that RC technology is underdeveloped, not mature or only used for research activities. While other EMC measurement environments like, for instance, open areas, (semi) anechoic rooms, etc. more or less have a clear, unique and standardized design, RCs have not such a unique definition. Again, this is not because we haven't yet found the proper one-and-only design, but rather it is mainly thanks to the ergodic hypothesis! All these (apparently) different stirring processes are essentially one, from the statistical physics point of view, if they individually satisfy the ergodic hypothesis. A substantial overview of RCs standardized applications and state-of-the-art is sketched in [49].

Having overviewed the extensive list of different possible stirring techniques should provide RC users, prospective or current ones, with valuable information in order to make proper design choices. Each stirring technique described in this paper features advantages and disadvantages, and which of them would better fit the case in particular depends on the specific application, available budget and space, set of requirements, etc. The fact that reverberation chamber design must often compromise between several factors such as, for instance, size of the chamber, desired frequency band of operation, size of the working volume, complexity of a particular stirring technique to be implemented, etc. is probably one of most direct ways to explain why this is such an active and prolific area of research.

How one should navigate and find a way through this maze of stirring technique choices? Not only the number of different stirring techniques is vast, moreover, each one of them often also comprise a labyrinth of choices on its own. For instance: where to place a stirrer? In which orientation? How many sources should I use? How to properly shake a tent? And so forth... One might even consider that some of these techniques would allow for a hybrid stirring strategy, combining two or more of those.

It might indeed feel like a difficult task to choose a proper stirring technique, having so many in the list to choose from. It might feel similar to what happens when looking at the menu in some restaurants, where each choice looks appealing.

Reverberation chambers à la carte.

Here's our menu. Enjoy!

References: 
[1] P. M. Morse, H. Feshbach: Methods of theoretical physics, Parts I and II, New York: McGrawHill, 1953.

[2] Electromagnetic compatibility (EMC) - Part 4-21: Testing and measurement techniques Reverberation chamber test methods, IEC 61000-4-21:2011.

[3] F. Monsef, A. Cozza, "Average Number of Significant Modes Excited in a Mode-Stirred Reverberation Chamber", IEEE Transactions on Electromagnetic Compatibility. 56(2):259-265, April 2014.

[4] Test method standard MIL-STD-1377: "Measurement of effectiveness of cable, connector, and weapon enclosure shielding and filters in precluding hazards of electromagnetic radiation to ordnance (HERO)", U. S. Department of defense, 20 August 1971.

[5] Paolini, E and Piccioli, L, "Measuring technique for assessing interference radiation produced from industrial, scientific and medical (I.S.M.) apparatus at microwaves," in Proc. IEEE Symp. Electromagn. Compat., New York, NY, USA, 1973, pp. 279-287.

[6] P. Corona and G. Latmiral: "Valutazione ed impegno normativo della camera reverberante dell'Istituto Universitario Navale", in Atti I Riunione Nazionale di Elettromagnetismo Applicato, L'Aquila, Rome, Italy, pp. 103-108, 1976.

[7] P. Corona, G. Latmiral, E. Paolini and L. Piccioli, "Use of reverberating enclosure for measurement of radiated power in the microwave range," IEEE Trans. on EMC., vol. 18, no. 2, pp. 54--59, May 1976.

[8] M. Migliaccio, G. Gradoni and L. R. Arnaut, "Electromagnetic Reverberation: The Legacy of Paolo Corona," in IEEE Transactions on Electromagnetic Compatibility, vol. 58, no. 3, pp. 643652, June 2016.

[9] M. Bäckström and O. Lundén, "Transmission cross sections of apertures measured by use of nested mode-stirred chambers," FOA Report, FOA-R-96-00359-3.2-SE, ISSN 1104-9154, Dec. 1996.

[10] J. Clegg, A. C. Marvin, J. F. Dawson, and S. J. Porter, "Optimization of stirrer designs in a reverberation chamber," IEEE Trans. Electromagn. Compat., vol. 47, no. 4, pp. 824-832, Nov. 2005.

[11] A. C. Marvin, J. F. Dawson, and J. Clegg, "Stirrer optimization for reverberation chambers," in Proc. Int. Symp. on Electromagnetic Compatibility. Eindhoven, The Netherlands: Technische Universiteit Eindhoven, 2004, pp. 330-335.

[12] Arnaut, L. R., "Operation of electromagnetic reverberation chambers and wave diffractors at relatively low frequencies"; IEEE Trans. on EMC, vo. 43 no 4 (Nov 2001), pp 637-653.

[13] Arnaut, L. R.; “Compound exponential distributions for undermoded reverberation chambers"; IEEE Trans EMC, vol 44 no 3 (Aug 2002), pp 442-457. 
[14] O. Lundén and M. Bäckström, "A factorial design of experiment for evaluation of mode stirrers in reverberation chambers," Proc IEEE Int. Symp. EMC, Istanbul, Turkey, Sep. 2003.

[15] Arnaut, L R; "Effect of size, orientation, and eccentricity of mode stirrers on their performance in reverberation chambers," IEEE Trans EMC, vol 48 no 3 (Aug 2006), pp 600-602.

[16] L. R. Arnaut, F. Moglie, L. Bastianelli, and V. Mariani Primiani, "Helical stirring for enhanced performance of reverberation chambers," IEEE Trans. Electromagn. Compat., vol. 59 (2017), DOI: 10.1109/TEMC.2016.2641386

[17] N.Wellander, O. Lundén, and M. Bäckström, "Experimental investigation and mathematical modeling of design parameters for efficient stirrers in mode-stirred reverberation chamber," IEEE Trans. Electromagn. Compat., vol. 49, no. 1, pp. 94-103, Feb. 2007.

[18] O. Lundén, N. Wellander, and M. Bäckström, "Stirrer blade separation experiment in reverberation chambers," in Proc. IEEE Int. Symp. Electromagn. Compat., Fort Lauderdale, FL, Jul. 2010, pp. 526-529.

[19] G. Bosco, C. Picciani, V. Mariani Primiani, and F. Moglie, "Numerical and experimental analysis of the performance of a reduced surface stirrer for reverberation chambers," in Proc. IEEE Int. Symp. Electromagn. Compat., Pittsburgh, PA, USA, 2012, pp. 156-161.

[20] D. Fedeli, M. Iualè, V. Mariani Primiani, and F. Moglie, "Experimental and numerical analysis of a carousel stirrer for reverberation chambers," in Proc. IEEE Int. Symp. Electromagn. Compat., Pittsburgh, PA, USA, 2012, pp. 228-233.

[21] F. Moglie and V. Mariani Primiani, "Numerical analysis of a new location for the working volume inside a reverberation chamber," IEEE Trans. Electromagn. Compat., vol. 54, no. 2, pp. 238-245, May 2012.

[22] Y. Huang, "The Investigation of Chambers for Electromagnetic Systems," D.Phil Thesis, University of Oxford, UK, 1993

[23] Frank B.J. Leferink, "High field strength in a large volume: the intrinsic reverberation chamber," IEEE Symposium on EMC, 1998, pp. 24-27

[24] Frank Leferink, Patent NL1010745, Test Chamber, 7-12-1998

[25] Frank Leferink, W.C. van Etten, "Optimal Utilization of a Reverberation Chamber", Euro EMC 2000, Symposium on EMC, Brugge, 2000, pp. 201-206, ISBN 90-76019-14-2

[26] Frank Leferink, W.C. van Etten, "Generating an EMC test field using a Vibrating Intrinsic Reverberation Chamber", EMC Society Newsletter, Spring 2001, pag. 19-25, ISSN 1089-0785.

[27] Arnaut, L. R. and West, P. D.; "Evaluation of the NPL Untuned Stadium Reverberation Chamber Using Mechanical and Electronic Stirring Techniques"; NPL Report CEM 11 (Aug 1998), ISSN 1369-6742, pp $1-176$. 
[28] Arnaut, L R; “Untuned 3D stadium reverberation chamber for microwave and millimeterwave frequencies"; Proc 1999 Mode-stirred Chamber, Anechoic Chamber and OATS Users Meeting (03-09 Jun 1999, Northbrook, IL), pp 1-6.

[29] Arnaut, L R and West, P D; “Electromagnetic reverberation near a perfectly conducting boundary"; IEEE Trans EMC, vol 48 no 2 (May 2006), pp 359-371.

[30] Arnaut, L R, Serra, R, and West, P D; "Statistical anisotropy in imperfect electromagnetic reverberation"; IEEE Trans EMC, vol 59 no 1 DOI: 10.1109/TEMC.2016.2606540

[31] P. Corona, "Comments and corrections," IEEE Trans. Electromagn. Compat., vol. 18, no. 4, p. 205, Nov. 1976.

[32] P. T. Jensen, A. P. Mynster, and R. B. Behnke, "Practical industrial EUT testing in reverb chamber: Experiences, findings and practical observations on high amplitude immunity testing of industrial equipment in reverberation chamber," in Proc. EMC Europe 2014 Int. Symp. Electromagn. Compat., Gothenburg, Sweden, Sep. 2014, pp. 274-279.

[33] Hatfield, M.O., "Shielding Effectiveness Measurements Using Mode-Stirred Chambers: A Comparison of Two Approaches", IEEE Transactions on EMC, Vol. 30, No. 3, p. 229-238, August 1988.

[34] Loughry, T.A., "Frequency Stirring: An Alternative Approach to Mechanical Mode-Stirring For The Conduct of Electromagnetic Susceptibility Testing", USAF Phillips Laboratory, PL-TR-911036, November 1991.

[35] M. L. Crawford, T. A. Loughry, M. O. Hatfield, G. J. Freyer, "Bandlimited, White Gaussian Noise Excitation for Reverberation Chambers and Applications to Radiated Susceptibility Testing", National Institute of Standards and Technology (NIST), Technical Note 1375, 1996.

[36] A. Cozza, "Probability Distributions of Local Modal-Density Fluctuations in an Electromagnetic Cavity", IEEE Trans. on EMC (2012), Vol. 54, no. 5, pp. 954-967.

[37] D.A. Hill, "Electronic mode stirring for reverberation chambers", IEEE Trans. on EMC (1994), Vol. 36, no. 4, pp. 294-299.

[38] A. Cozza, W.J. Koh, Y.S. Ng, Y.Y. Tan, "Controlling the state of a reverberation chamber by means of a random multiple-antenna stirring", 2012 Asia-Pacific Symposium on Electromagnetic Compatibility.

[39] E. Voges, T. Eisenburger: "Electrical Mode Stirring in Reverberating Chambers by Reactively Loaded Antennas", IEEE Transactions on Electromagnetic Compatibility, vol. 49, no. 4, pp. 756761, November 2007.

[40] Y. Huang and D. J. Edwards, "A novel reverberating chamber: source-stirred chamber," IEE 8th International Conference on Electromagnetic Compatibility, pp.120-124, Edinburgh, UK, September 1992. 
[41] S. Boyes and Y. Huang, Reverberation Chambers - theory and applications to EMC and Antenna Measurements, John Wiley \& Sons, London, 2016

[42] G. Cerri, V. Primiani, S. Pennesi, and P. Russo, "Source stirring mode for reverberation chambers," IEEE Transactions on Electromagnetic Compatibility, vol. 47, no. 4, pp. 815 - 823, Nov. 2005.

[43] G. Cerri, V. M. Primiani, C. Monteverde and P. Russo, "A theoretical feasibility study of a source stirring reverberation chamber," IEEE Transactions on Electromagnetic Compatibility, vol. 51, pp.3 - 11, Feb. 2009

[44] C. Monteverde, G. Koepke, C. Holloway, J. Ladbury, D. Hill, V. Primiani, and P. Russo, "Source stirring technique for reverberation chambers; experimental investigation," International Symposium on Electromagnetic Compatibility - EMC Europe 2008, Sept. 2008.

[45] J. Kunthong and C. Bunting, "Source-stirring and mechanical-stirring reverberation chamber measurement comparison for $900 \mathrm{MHz}$ and $1800 \mathrm{MHz}$," 2009 IEEE International Symposium on Electromagnetic Compatibility, pp. 193 - 196. Aug. 2009.

[46] E. Amador and P. Besnier, "A source stirring analysis in a reverberation chamber based on modal expansion of the electric field," 2015 IEEE International Symposium on EMC, Sept. 2015

[47]

http://www.haier.com/cn/consumer/small applications/cyxjd/wbl/201107/t20110710 24172.s $\underline{\text { html }}$

[48] M. Klingler: Dispositif et procédé de brassage électromagnétique dans une chambre réverbérante à brassage de modes - Patent FR 2887 337, 17/06/2005.

[49] L.R. Arnaut, "Mode-stirred reverberation chambers: A paradigm for spatio-temporal complexity in dynamic electromagnetic environments", Wave Motion, Vol. 51, Issue 4, June 2014, Pages 673-684. 